\title{
A non-ideal MHD Gadget: Simulating massive galaxy clusters
}

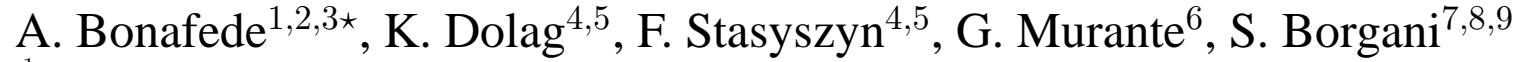 \\ ${ }^{1}$ Jacobs University Bremen, Campus Ring 1, D-28759 Bremen, Germany \\ ${ }^{2}$ INAF Istituto di Radioastronomia, via P. Gobetti 101, I-40129 Bologna, Italy \\ ${ }^{2}$ Università di Bologna, Dip. di Astronomia, via Ranzani 1, I-40126 Bologna, Italy \\ ${ }^{4}$ Universitätssternwarte München, München, Germany \\ ${ }^{5}$ Max-Planck-Institut für Astrophysik, Garching, Germany \\ ${ }^{6}$ INAF - Osservatorio astronomico di Torino, Str. Osservatorio 25, I-10025, Pino Torinese, Torino, Italy \\ ${ }^{7}$ Universitá di Trieste, Dip. di Fisica, Sezione di Astronomia, via Tiepolo 11, I-34131 Trieste, Italy \\ ${ }^{8}$ INAF - Osservatorio astronomico di Trieste, via Tiepolo 11, I-34131 Trieste, Italy \\ ${ }^{9}$ INFN - Istituto nazionale di Fisica nucleare, Trieste, Italy
}

Accepted ???. Received ???; in original form ???

\begin{abstract}
Magnetic fields in the intra-cluster medium of galaxy clusters have been studied in the past years through different methods. So far, our understanding of the origin of these magnetic fields, as well as their role in the process of structure formation and their interplay with the other constituents of the intra-cluster medium is still limited. In the next years the up-coming generation of radio telescopes is going to provide new data that have the potential of setting constraints on the properties of magnetic fields in galaxy clusters.

Here we present zoomed-in simulations for a set of massive galaxy clusters $\left(M_{v} \geqslant\right.$ $10^{15} h^{-1} M_{\odot}$ ). This is an ideal sample to study the evolution of magnetic field during the process of structure formation in detail. Turbulent motions of the gas within the ICM will manifest themselves in a macroscopic magnetic resistivity $\eta_{m}$, which has to be taken explicitly into account, especially at scales below the resolution limit. We have adapted the MHD GADGET code by Dolag \& Stasyszyn (2009) to include the treatment of the magnetic resistivity and for the first time we have included non-ideal MHD equations to better follow the evolution of the magnetic field within galaxy clusters. We investigate which value of the magnetic resistivity $\eta_{m}$ is required to match the magnetic field profile derived from radio observations. We find that a value of $\eta_{m} \sim 6 \times 10^{27} \mathrm{~cm}^{2} \mathrm{~s}^{-1}$ is necessary to recover the shape of the magnetic field profile inferred from radio observations of the Coma cluster. This value agrees well with the expected level of turbulent motions within the ICM at our resolution limit. The magnetic field profiles of the simulated clusters can be fitted by a $\beta$-model like profile (Cavaliere \& Fusco-Femiano 1976), with small dispersion of the parameters. We find also that that the temperature, density and entropy profiles of the clusters depend on the magnetic resistivity constant, having flatter profiles in the inner regions when the magnetic resistivity increases.
\end{abstract}

Key words: (magnetohydrodynamics)MHD - magnetic fields - methods: numerical - galaxies: clusters

\section{INTRODUCTION}

Magnetic fields are an important ingredient to understand the physical processes taking places in the intra-cluster medium (ICM) of galaxy clusters. Their presence is demonstrated by radio observations, which, since the last 30 years, have revealed diffuse and faint radio sources filling the central $\mathrm{Mpc}^{3}$ of some galaxy clusters (radio halos, see e.g. Giovannini et al. 2009; Venturi et al. 2008). These sources arise because of the interaction of highly relativistic electrons with the ICM magnetic fields. About 30 radio halos

\footnotetext{
* E-mail: a.bonafede@jacobs-university.de
}

are known so far, and all of them are found in clusters with clear signatures of on-going or recent merger activity (e.g. Buote 2001; Govoni et al.2001; Cassano et al.2010). The origin of the relativistic particles still needs to be understood, although several models have been proposed. Shocks and turbulence associated with merger events are expected to inject a considerable amount of energy in the ICM, that could compress and amplify the magnetic field and (re-)accelerate relativistic electrons, giving thus rise to the observed radio emission (see Ferrari et al. 2008; Dolag et al.2008, for recent reviews of the subject). Understanding the magnetic field amplification and evolution during the process of structure formation is then mandatory for modeling the acceleration, transport and inter- 
actions of non-thermal energetic particles and thus to understand the observed emission. In addition, an accurate modeling of the magnetic field properties is necessary to understand both the heat transport and the dissipative processes in the ICM.

The properties of magnetic fields in the ICM have been investigated in the past through cosmological simulations, performed with different numerical codes (Dolag et al. 1999, 2002, 2005; Dubois \& Teyssier 2008; Dolag \& Stasyszyn 2009; Collins et al. 2010) and also through Faraday Rotation measures analysis (e.g. Murgia et al. 2004; Govoni et al. 2006; Vogt \& Enßlin 2005; Laing et al. 2008; Bonafede et al. 2010). The comparison with observed data is necessary to constrain the main magnetic field properties, and it is starting now to be feasible thanks to the progress that has been done in the recent years. One key aspect is that, so far, large scale radio emission is mainly detected in very massive clusters. Such massive systems are not easily studied by numerical simulations, since the size of the density fluctuations responsible for the formation of massive halos is large, i.e. $\sim 20 h^{-1} \mathrm{Mpc}$, and the value of the cosmological parameter $\sigma_{8}$ in the standard $\Lambda C D M$ model requires that statistically a total volume of $\sim 200 h^{-1} M p c^{3}$ needs to be sampled by simulations in order to produce at least one cluster as massive as $\sim 10^{15} h^{-1} M_{\odot}$. An important step for studying non-thermal phenomena is to perform simulations based on extremely large cosmological volumes, e.g. $1 \mathrm{Gpc}$ side-length. Such large volumes cannot be simulated at the resolution reached by observations, so that re-simulation techniques have been developed (e.g.GRAFIC Bertschinger 1995; ZIC Tormen et al. 1997; Jenkins 2010). When such high resolution is reached, the physic of the baryonic component must be followed with particular care. The magnetic field amplification, in particular, depends on the small scale motions of the gas. Hence, as the resolution increases smaller scale motions are revealed, and the magnetic field amplification increases accordingly (see e.g. Dolag et al. 2008).

In this paper we present a set of galaxy clusters extracted by a low resolution DM simulation and re-simulated at high resolution (the softening length is $\sim 5 \mathrm{kpc} h^{-1}$ ) within a cosmological framework in order to resolve scales comparable to those reached by observations. This work is focused on the 24 most massive galaxy clusters $\left(M_{200}>10^{15} h^{-1} \mathrm{Mpc}\right)$ of our sample. Simulations are performed for the first time relaxing the assumption of ideal MHD, and including a resistivity term in the induction equation $\left(\eta_{m}\right)$. Our sample of simulated galaxy cluster is publically available for further studies 1 . In this paper we present the simulated cluster sample: the MHD implementation with some test problems (Section.2), the re-simulation technique used (Section. 3 and more detailed in the appendix); the effect of different values for $\eta_{m}$ are analyzed and discussed in Section 4 where the main properties of the clusters are also presented. Finally, discussion and conclusions are reported in Section 5

This is a first paper aimed at presenting the cluster sample, the zoom-in initial conditions, and the non-ideal MHD implementation in the GADGET code. This sample has also been used by Fabjan et al. (2011) for a study of the scaling relations of X-ray mass proxies. In a future paper the authors will investigate in more detail the cluster properties, and the interplay between thermal and non-thermal components in the ICM.

${ }^{1}$ contact a.bonafede@jacobs-university.de or kdolag@mpagarching.mpg.de

\section{NON-IDEAL MHD SIMULATIONS}

Within the last decade, cosmological simulations of structure formation have shown that the observed properties of magnetic fields in galaxy clusters are direct consequences of turbulent amplification driven by the the structure formation process (Dolag et al. 1999, 2002, 2005; Brüggen et al. 2005; Dubois \& Teyssier 2008; Dolag \& Stasyszyn 2009; Collins et al. 2010). Simulations performed with different codes reach good agreement in predicting that the ratio of the bulk kinetic energy to the thermal energy has an upper limit of $\sim 10-20 \%$ (see e.g. the review by Borgani \& Kravtsov 2009, and references therein). Recently, noncosmological MHD simulations of merging galaxies (Kotarba et al. 2009, 2010) predict that the magnetic field is amplified up to a level close to $\sim 10-20 \%$ of the thermal energy. The same is expected for the ICM of galaxy clusters. Although the properties of magnetic fields in galaxy clusters are still not strongly constrained from the observational point of view, present data suggest that the magnetic field energy content is not amplified up to the level of the kinetic energy. In the Coma cluster, for example, the turbulent energy content is $\sim 10 \%$ of the thermal one (Schuecker et al. 2004), whereas the magnetic energy content associated within the observed magnetic field of $4.7 \mu G$ (Bonafede et al. 2010) is only $1.6 \%$ of the thermal one. Dissipative processes could possibly explain the saturation of magnetic fields far below the level of the kinetic energy. Such dissipative processes, driven by the physical properties of the ICM plasma, are not investigate in numerical simulations so far, but Dolag \& Stasyszyn (2009) have shown that dissipative processes driven by numerical diffusivity may alter the central properties of the magnetic field profiles obtained by numerical simulations.

The simulations we present in this paper were carried out with GADGET-3 (Springel 2005), the current version of the parallel TreePM+SPH simulation code GADGET (Springel et al. 2001). It includes an entropy-conserving formulation of SPH (Springel \& Hernquist 2002), the implementation of ideal MHD (Dolag \& Stasyszyn 2009) and an implementation of a divergence cleaning scheme (Dolag \& Stasyszyn 2009; Børve et al. 2001). The cosmological simulations presented here assume an initially homogeneous magnetic field of $10^{-12} \mathrm{G}$ co-moving.

In previous works it was usually assumed that the electric conductivity of the gas is infinite, meaning that the second term of the induction equation (Eq. 1) vanishes $\left(\eta_{m}=0\right)$.

$\frac{\partial \vec{B}}{\partial t}=\vec{\nabla} \times(\vec{v} \times \vec{B})+\vec{\nabla} \times\left(\eta_{m} \vec{\nabla} \times \vec{B}\right)$.

This assumption results in a magnetic field frozen into the gas. We have extended the treatment of the induction equation to cover the resistive MHD equation. Here we will assume for simplicity a spatially constant resistivity term $\eta_{m}$. In Section 4.2 the physical origin of this term is analyzed and the assumption will be discussed. Under the constraint $\vec{\nabla} \cdot \vec{B}=0$, and $\eta_{m}$ spatially constant, the induction equation for resistive MHD can then be written as:

$\frac{\partial \vec{B}}{\partial t}=(\vec{B} \cdot \vec{\nabla}) \vec{v}-\vec{B}(\vec{\nabla} \cdot \vec{v})+\eta_{m} \vec{\nabla}^{2} \vec{B}$.

The resistivity dependent terms have been implemented in the code following the approach adopted for the artificial dissipation by Price \& Monaghan (2004a b, 2005). In particular, we refer to Dolag \& Stasyszyn (2009) where the artificial dissipation term has been implemented in the GADGET code. More specifically, the resistive term is included in the induction equation as 


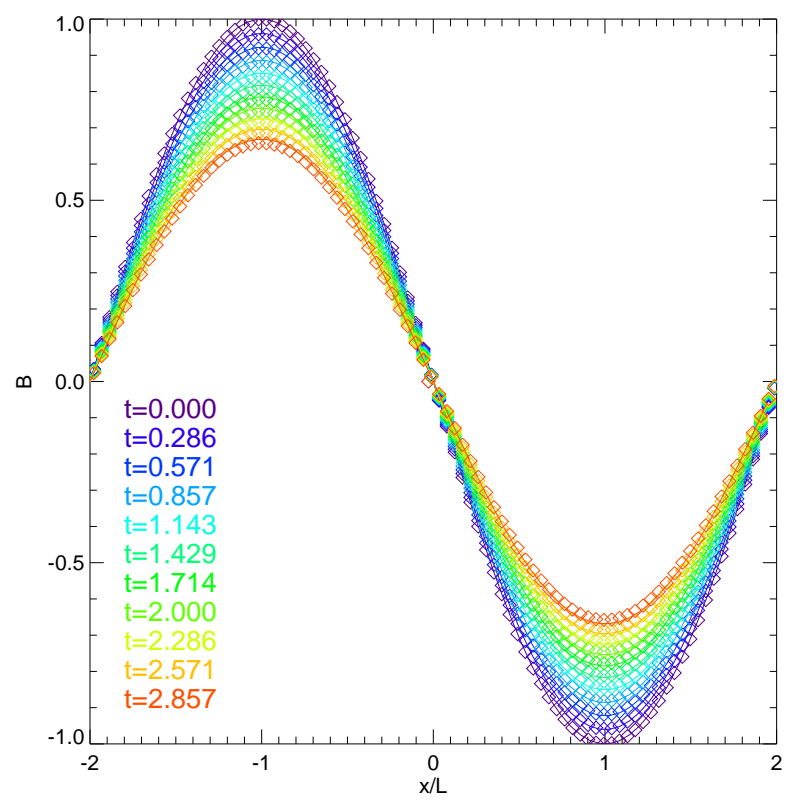

Figure 1. Comparison of the results from the simulations (diamonds) to the analytic solution (lines) at different output times. The magnetic resistivity $\eta_{m}$ was set to 1 in this test. For graphical reasons, only one diamond each 8th particle in $\mathrm{x}$-direction was plotted for every time step.

$\left.\frac{\partial \vec{B}_{i}}{\partial t}\right|_{r e s}=\frac{\eta_{m} \rho_{i}}{H a^{2}} \sum_{j=1}^{N} \frac{m_{j}}{\hat{\rho}_{i, j}^{2}}\left(\overrightarrow{B_{i}}-\overrightarrow{B_{j}}\right) \frac{\overrightarrow{r_{i, j}}}{\left|\overrightarrow{r_{i, j}}\right|} \cdot \vec{\nabla}_{i} W_{i}$

Where $i$ and $j$ refer to two generic particles in the simulation, $\vec{r}_{i, j}$ is the distance between particle $i$ and $j, W$ is the SPH kernel, and the factor $\left(H a^{2}\right)^{-1}=\frac{d t}{d a}$ takes into account that the internal time variable in GADGET is the expansion parameter $a$. The resistivity term implemented in the induction equation causes a change in the Entropy $A$ at the rate

$\left.\frac{d A_{i}}{d t}\right|_{r e s}=-\frac{\gamma-1}{2 \mu_{0} \rho_{i}^{\gamma-1}} \sum_{j=1}^{N} \frac{m_{j}}{\hat{\rho}_{i, j}^{2}}\left(\vec{B}_{i}-\vec{B}_{j}\right)^{2} \frac{r_{i, j}}{\left|r_{i, j}\right|} \cdot \vec{\nabla}_{i} \bar{W}_{i, j}$,

where $\bar{W}_{i, j}$ indicates the mean between the two kernels $W_{i}$ and $W_{j}, \gamma$ is the adiabatic index of the gas. We refer to Dolag \& Stasyszyn (2009) for more details about the numerical implementation. Since we basically replaced the artificial dissipation already implemented and tested (Dolag \& Stasyszyn 2009) with a physically motivated magnetic resistivity, the only tests that are left to be performed to validate the numerical scheme are those regarding the ability of the code to reproduce the correct dissipation timescale. This can be done, in the case of a spatially constant $\eta_{m}$, by investigating the magnetic field evolution for simple test problems.

\subsection{Test 1: A one-dimensional slab in a 3D setup}

We consider first the time evolution of a one-dimensional magnetic field $(\vec{B}=B(t) \hat{y})$ in a one dimensional slab at rest having side length $4 L$. In order to test the code within the configuration used for cosmological simulations, we performed the test in a $3 \mathrm{D}$ setup using a glass-like particle distribution and solving a planar test problem within this 3D setup (White 1996). We started with

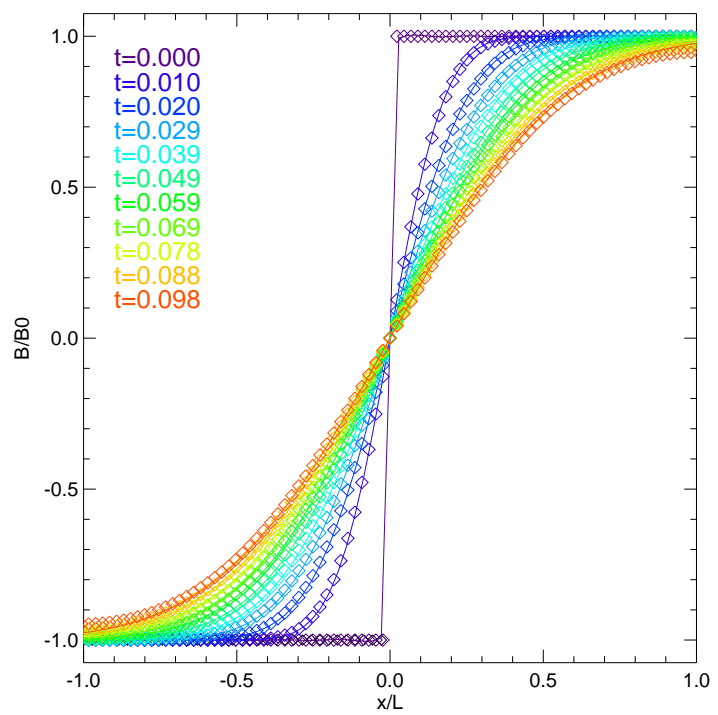

Figure 2. Comparison of the results from the simulations (diamonds) to the analytic solution (lines) at different output times. The magnetic resistivity $\eta_{m}$ was set to 1 in this test. For graphical reasons 1 diamonds each 4th particle in $\mathrm{x}$-direction is plotted for every time.

$700 \times 10 \times 10$ particles, having a mean inter-particle separation along the $\mathrm{x}$ axis of $5.7 \times 10^{-3} \mathrm{~L}$. Using 64 neighbors within the SPH interpolant this correspond to roughly 35 resolution elements per length $L$.

The induction equation here reduces to

$\frac{\partial B}{\partial t}=\eta_{m} \frac{\mathrm{d}^{2} B}{\mathrm{~d} x^{2}}$

which has the analytical solution:

$B(t)=\exp \left(-\eta_{m} t\left(\frac{2 \pi}{4 L}\right)^{2}\right) B_{0} \sin \left(\frac{2 x \pi}{4 L}\right)$.

We set $B_{x}=B$ and $B_{y}=B_{z}=0$, and followed the evolution of an initial magnetic field $B=B_{0} \sin (2 \pi x /(4 L))$.

In Figure 1 the time evolution of the system is shown. The results obtained from the numerical simulation (diamonds) are compared with the analytic solution (lines) for various time steps, showing an excellent agreement.

\subsection{Test 2: Magnetic diffusion across a step in a 3D setup}

As a second test, we consider here a one dimensional slab. The magnetic field is described by $\vec{B}=B(x, t) \hat{y}$, and a step profile for the magnetic field was included according to:

$B(x, 0)= \begin{cases}+B_{0} & x>0 \\ -B_{0} & x<0\end{cases}$

As in the previous test, the simulation was performed in a full, three dimensional setup using a glass-like particle distribution and solving a planar test problem within this $3 \mathrm{D}$ setup. We started with 700x10x10 particles, having a mean inter-particle separation along the $\mathrm{x}$ axis of $5.7 \times 10^{-3} \mathrm{~L}$. Using 64 neighbors within the SPH interpolant this correspond to roughly 35 resolution elements per length $L$. Under the constrain

$B(-L, t)=-B(L, t)=B_{0}$, 
meaning that the magnetic field is held fixed at two points $( \pm L)$ the solution of the diffusion equation can be written as

$B(x, t)=B_{0} \frac{x}{L}+\frac{2 B_{0}}{\pi} \sum_{n=1}^{\infty} \frac{1}{n} \exp \left(\frac{-n^{2} \pi^{2} \eta_{m} t}{L^{2}}\right) \sin \left(\frac{n \pi x}{L}\right)(9)$

(see Wilmot-Smith et al. 2005). In Figure 2 the results of the numerical simulation (diamonds) are compared to the analytic solution (lines) at different time steps, as reported in the figure panel. The magnetic field diffuses rapidly and converges towards the steady-state solution, $B(x)=B_{0}(x) / L$. Since we have not implemented the necessary boundary conditions to keep $B=B_{0}$ fixed at the borders, this simulation was stopped early.

\subsection{Total energy conservation}

The two tests described in Section. 2.1 and 2.2 demonstrate the ability of the code to correctly solve the diffusion equation. When real physical problems are considered, the magnetic energy dissipated is explicitely added to the energy equation, i.e. it is transferred to the system, so that the total energy is conserved. Hence, within the cosmological simulations presented in the following Sections, the energy of the dissipated magnetic field is transferred into heat. This energy is added explicitly to the internal energy, similarly to what is done when artificial magnetic resistivity is used as a regularization scheme to suppress numerical instabilities (Price \& Monaghan 2007, Dolag \& Stasyszyn 2009). We refer to Dolag \& Stasyszyn (2009) for the details of the numerical implementation, and in particular, to section 3.1 and Figure 4 (upper middle panel) of Dolag \& Stasyszyn (2009) where such issues are discussed and analyzed. In the two tests presented in Section 2.1 and 2.2 the conversion of the dissipated magnetic energy into heat has been switched off, since the solution we compare with do not include such conversion.

\section{CONSTRUCTING THE CLUSTER SET}

\subsection{The parent simulation}

The clusters were selected from a N-body cosmological simulation performed according to a flat $\Lambda \mathrm{CDM}$ cosmological model, with $\Omega_{m}=0.24$ (the matter density parameter), $\Omega_{b a r}=0.04$ (the contribution given by baryons), $h=0.72$, and $\sigma_{8}=0.8$. The power spectrum for the primordial density fluctuations $P(k) \propto k^{n}$ is characterized by $n=0.96$. This simulation was carried out with the massively parallel TREE+SPH code GADGET-3, the new version of the GADGET code (Springel et al. 2001; Springel 2005) and consists of a periodic box of size $1 \mathrm{Gpc} h^{-1}$. The cluster identification was performed at $z=0$ using a standard Friend of friends algorithm (Davis et al. 1985). The linking length was fixed to 0.17 the mean inter-particle separation between DM particles, corresponding to the virial over-density in the adopted cosmological model. This large simulated cosmological box contains 64 clusters with $M_{F O F}>10^{15} h^{-1} M_{\odot}$ at $z=0$. Hence, it represents a proper sample to study the general properties of massive galaxy clusters. Since we want to analyze the magnetic field properties, and compare our results with those found from Coma cluster observations, a statistical set of galaxy clusters with masses similar to the one of Coma is needed. Note that up to now, no such sample of high resolution re-simulations of massive galaxy clusters has been constructed.
Table 1. Properties of the high mass cluster set.

\begin{tabular}{|c|c|c|c|c|}
\hline Cluster & $\begin{array}{c}M_{\text {vir }} \\
{\left[10^{15} h^{-1} M_{\odot} h_{72}^{-1} M_{\odot}\right]}\end{array}$ & $\begin{array}{c}R_{\mathrm{vir}} \\
\operatorname{kpc} h^{-1}\end{array}$ & $\begin{array}{c}L_{X} \\
{\left[10^{45}\right] \operatorname{erg} s^{-1}}\end{array}$ & $\begin{array}{l}T_{M W} \\
{[\mathrm{keV}]}\end{array}$ \\
\hline D_1 & $1.62-2.25$ & 2521 & 4.10 & 6.1 \\
\hline D_2 & $1.51-2.09$ & 2442 & 3.60 & 5.0 \\
\hline D_3 & $1.47-2.04$ & 2430 & 6.30 & 6.0 \\
\hline D_4 & $1.50-2.09$ & 2438 & 4.40 & 3.8 \\
\hline D_5 & $1.53-2.12$ & 2455 & 5.34 & 5.6 \\
\hline D_6 & $1.23-1.70$ & 2271 & 1.89 & 5.1 \\
\hline D_7 & $1.78-2.47$ & 2585 & 3.15 & 6.2 \\
\hline D_8 & $1.85-2.57$ & 2707 & 2.58 & 5.6 \\
\hline D_9 & $1.68-2.33$ & 2549 & 5.06 & 6.2 \\
\hline D_10 & $1.74-2.42$ & 2569 & 4.60 & 6.6 \\
\hline D_11 & $3.09-4.29$ & 3133 & 10.5 & 8.7 \\
\hline D_12 & $1.68-2.32$ & 2537 & 2.01 & 4.7 \\
\hline D_13 & $1.17-1.63$ & 2247 & 2.45 & 5.6 \\
\hline D_14 & $1.56-2.16$ & 2484 & 4.87 & 5.5 \\
\hline D_15 & $1.88-2.61$ & 2647 & 4.95 & 5.6 \\
\hline D_16 & $1.40-1.93$ & 2382 & 8.10 & 8.0 \\
\hline D_17 & $1.81-2.51$ & 2626 & 8.95 & 8.4 \\
\hline D_18 & $1.38-1.92$ & 2366 & 6.61 & 6.9 \\
\hline D_19 & $1.30-1.81$ & 2346 & 7.21 & 6.6 \\
\hline D_20 & $1.07-1.49$ & 2165 & 1.86 & 3.5 \\
\hline D_21 & $1.61-2.23$ & 2507 & 4.95 & 5.6 \\
\hline D_22 & $1.67-2.32$ & 2536 & 3.75 & 5.9 \\
\hline D_23 & $1.90-2.63$ & 2648 & 7.43 & 7.9 \\
\hline D_24 & $1.59-2.21$ & 2490 & 2.5 & 4.9 \\
\hline \multicolumn{5}{|c|}{ Col. 1: Cluster name; Col. 2: Total mass inside $R_{\mathrm{vir}}$; } \\
\hline \multicolumn{5}{|c|}{ Col. 3: Virial radius; } \\
\hline \multicolumn{5}{|c|}{ Col 4: Estimated X-ray Luminosity in the band $0.1-10 \mathrm{keV}$; } \\
\hline Col 5: Mea & mperature (mass weighted); & & & \\
\hline
\end{tabular}

\subsection{Cluster selection and Initial Conditions}

Clusters were selected from the parent simulation on the basis of their mass only. We selected the 24 most massive objects among those with $M_{F O F}>10^{15} h^{-1} M_{\odot}$ and re-simulated each of these clusters at higher resolution by using the Zoomed Initial Conditions code (ZIC, Tormen et al. 1997). In the appendix the iterative procedure used to obtain the high resolution initial conditions is described in detail. The setup of initial conditions was optimized to guarantee a spherical volume around each cluster with radius of $\sim$ 5-6 virial radii $\left(R_{\mathrm{vir}}\right)$ simulated at high resolution (HR region) and free of contamination by low resolution, boundary particles. Two of the cluster initially selected by the Friend of Friends algorithm turned out to have a companion with mass $>10^{15} h^{-1} M_{\odot}$. Other systems are undergoing a merger event at $z=0$ with less massive companions. In addition, other clusters with masses between $10^{14} h^{-1} M_{\odot}$ and $10^{15} h^{-1} M_{\odot}$ where found in the HR region of the main targets. 50 of them are cleaned by low resolution particles inside their virial radius. Therefore, the final sample consists of 76 clusters with masses larger than $10^{14} h^{-1} M_{\odot}$, comprising both isolated and merging systems. The massive cluster set is shown in Table 2.3 In the Appendix (Table A1 more details about the cluster surroundings are given, while in Table $\mathrm{A} 2$ the clusters with $M>10^{14} h^{-1} M_{\odot}$, found within $5 R_{\mathrm{vir}}$ from the massive targets, are listed. They are simulated at high resolution up to 1-5 $R_{\mathrm{vir}}$, as reported in that Table.

The virial mass of each cluster was defined as the mass contained within a radius encompassing an average density equal to the virial density, $\rho_{v i r}$, predicted by the top-hat spherical collapse model. For the assumed cosmology it is $\rho_{v i r}=95 \rho_{c}$, where $\rho_{c}$ is the critical 


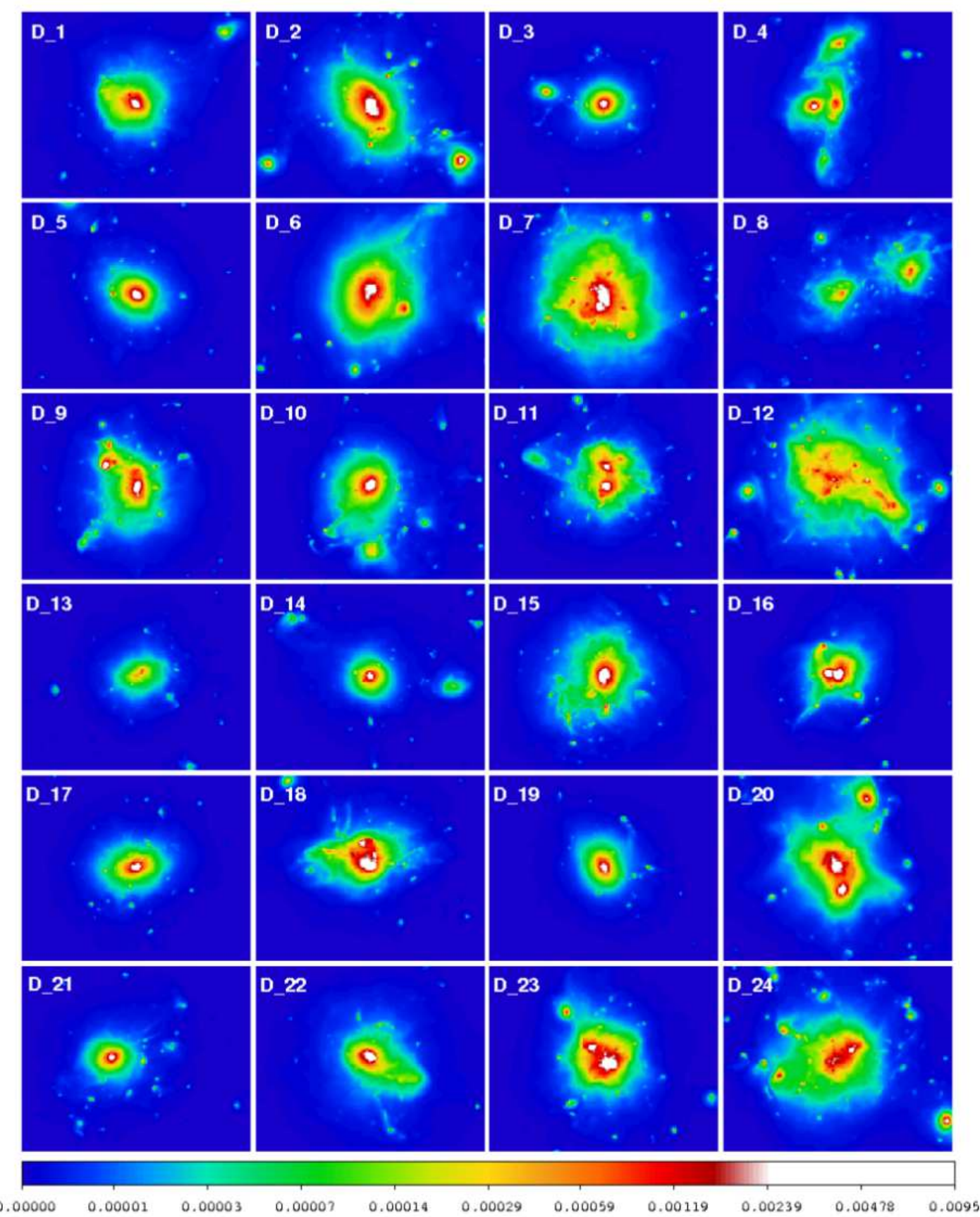

Figure 3. Projected X-ray surface brightness of the clusters in our sample computed in the range $0.1-10 \mathrm{keV}$ (square root scale). The side of each box corresponds to $\sim 1.6 \times 1.4 R_{\text {vir }}$.

cosmic density (Eke et al. 1996). In this work we focus onto the 24 originally selected clusters, as they represent a statistical well defined, volume and limited sample of massive clusters.

Once the ICs for the DM components have been obtained, gas particles were added (see appendix for details). The mass of DM and gas particle is $0.84 \times{ }^{9} h^{-1} M_{\odot}$ and $0.16 \times 10^{9} h^{-1} M_{\odot}$ respectively. The gravitational softening length used is $5 \mathrm{kpc} h^{-1}$, which corresponds to the smallest SPH smoothing length reached in the dense cluster centers.

\subsection{The high mass cluster set}

Simulations of these cluster set including radiative losses and star-formation are presented in Fabjan et al. (2011). Here, we focus on non-radiative simulations, since our aim is to study the effect of the magnetic field, and of non-ideal MHD.

From the final snapshots of these simulations we derived the projected X-ray surface brightness images, by using a map-making algorithm (Dolag et al. 2005). The predicted emission of every $\mathrm{SPH}$ particle is projected along the line of sight considering an 
integration depth of $\pm 5 R_{\mathrm{vir}}$ around the center of simulated clusters. The X-ray Luminosity $\left(L_{X}\right)$ is computed in the range $0.1-10 \mathrm{keV}$. The $\mathrm{X}$-ray surface brightness images of the clusters are shown in Figure 3 The values of $L_{X}$ and of the gas temperature inside the virial radius are reported in Table 2.3 Clusters in different dynamical state belong to this sample and consequently the X-ray surface brightness images show quite different morphologies. Several clusters are disturbed in the very internal part, indicating that a merger event has just occurred (e.g.D_12), while other clusters have multiple peaks in the X-ray images, like e.g.D_4. Some clusters appear to have a regular shape, and others are going to interact with a smaller halo, that is visible in the X-ray images (e.g.D_1). In the sample we also found an ongoing merger event between two massive clusters (D_8, interacting with another cluster of $M>10^{15} h^{-1} M_{\odot}$ ). We note that the over all range of morphologies found in this mass limited sample compares qualitatively well with complete, observed samples (like the REXCESS sample, Böhringer et al. 2007), where also such extremely perturbed systems are found).

\section{MAGNETIC FIELDS IN MASSIVE CLUSTERS}

The properties of the ICM magnetic fields start now to be better understood, thanks to an increasing effort in analyzing Faraday Rotation Images of sources located either inside clusters and in their background (e.g.Murgia et al. 2004, Clarke 2004, Johnston-Hollitt et al. 2004, Vogt \& Enßlin 2005 Govoni et al. 2006, Bonafede et al. 2010). In general, the magnetic field in clusters inferred from these observations is found to be consistent with a magnetic field driven by the turbulence within the ICM and generally shows a radial decline. Once the density profile $\rho(r)$ has been inferred from X-ray observations, the magnetic field profile in galaxy clusters is supposed to follow the gas density profile according to:

$B(r)=B_{0} \rho(r)^{\alpha}$.

The fluctuations within the magnetic field are usually modeled assuming a power-law power spectrum, described by a slope $\eta$, a maximum length scale $\Lambda_{\max }$ (which can be related to the outer scale of the turbulence in within the ICM) and a minimum length scale $\Lambda_{\min }$ (which in case it is resolved, could be related to dissipative scales, either viscous or resistive). These model parameter are inferred by comparing the expected Rotation Measure statistics (mean, dispersion, auto-correlation function and structure function) and the polarization properties of the radio galaxies to the observed ones. So far the magnetic field in the Coma cluster is the one that is best constrained. It has been inferred from RM observations of seven radio-sources located at projected distances of 50 to $1500 \mathrm{kpc}$ from the cluster center. The best fit model results to be the one with $B_{0}=4.7_{-0.8}^{+0.7} \mu G, \alpha=0.5_{-0.1}^{+0.2}$, and $\Lambda_{\min } \sim 2$ kpc (Bonafede et al. 2010). Although previous cosmological MHD simulations of galaxy clusters produced magnetic field configuration which lead to Rotation Measure statistics similar to the observed ones (Dolag et al. 1999, 2002, 2005), the magnetic field profile tended to be steeper, with $\alpha \sim 1$ (Dolag et al. 2001). In addition, the values of the central magnetic field obtained from high-resolution simulations resulted to be slightly larger than observed (Donnert et al. 2009), but it was noticed that the magnetic field profiles are significantly altered if the underlying numerical

\begin{tabular}{cccc}
\hline$\eta_{T}\left[\mathrm{~cm}^{2} \mathrm{~s}^{-1}\right]$ & $\eta_{m}$ & $v_{\text {turb }}\left[\mathrm{kms}^{-1}\right]$ & $\lambda_{\text {turb }}[\mathrm{kpc}]$ \\
$1.5 \times 10^{27}$ & 5 & 25 & 2 \\
$3 \times 10^{27}$ & 10 & 50 & 2 \\
$6 \times 10^{27}$ & 20 & 50 & 4 \\
\hline
\end{tabular}

Table 2. Diffusion coefficients used in our simulations (in internal and physical units) together example values of turbulent length-scales and velocities which would correspond to such values.

\begin{tabular}{ccc}
\hline$\eta_{\text {turb }}\left[\mathrm{cm}^{2} \mathrm{~s}^{-1}\right]$ & Process & ref. \\
$3-5 \times 10^{28}$ & CR propagation (value at $1 \mathrm{GeV})$ & 1 \\
$3 \times 10^{25}$ & CR driven dynamo in galaxies & 2 \\
$1 \times 10^{29}$ & powering the Coma radio halo & 3 \\
$2 \times 10^{27}$ & turbulent cascade observed in Coma at $2 \mathrm{kpc}$ & 4 \\
$2 \times 10^{28}$ & turbulent cascade from simulations at $7.8 \mathrm{kpc}$ & 5 \\
$6 \times 10^{27}-4.5 \times 10^{29}$ & iron abundance profile in clusters & 6 \\
\hline
\end{tabular}

Table 3. Values of the diffusion coefficients commonly used in the literature, and observationally inferred. References are: 1: Strong et al. (2007), 2: Lesch \& Hanasz (2003), 3: Schlickeiser et al. (1987), 4: Schuecker et al. (2004), 5: Maier et al. (2009), 6: Rebusco et al. (2006). See text for details.

MHD implementation suffers from the presence of numerical diffusion (Dolag \& Stasyszyn 2009).

\subsection{Testing the effect of the magnetic resistivity}

Having a stable numerical scheme at hand, which does not suffer from numerical diffusion outside the SPH smoothing length (Stasyszyn 2011, in preparation), we can investigate for the first time the role of a physically motivated resistivity $\eta_{m}$ in shaping the ICM magnetic field profile. From our set of massive clusters, which have all masses comparable to the Coma's one, we selected four objects that at $z=0$ show X-ray morphologies similar to the one of Coma. In particular, we avoid selecting clusters with very spherical morphology as well as clusters with clear multiple X-ray brightness peaks. Figure 4 shows the X-ray morphology of the 4 selected clusters for the 3 spatial projection directions. This sub-set of clusters has been simulated with different value of $\eta_{m}$, with the aim of studying the resulting shape and central value of the magnetic field. Figure 4 shows the magnetic field profiles of those clusters compared to the best fit model for the Coma cluster, encompassed by the $\pm 3 \sigma$ region. Whereas all magnetic field profiles obtained from the simulations are within the $3 \sigma$ region in the outer parts, the profiles with small magnetic diffusion $\left(\eta_{m}=1.5 \times 10^{27} \mathrm{~cm}^{2} \mathrm{~s}^{-1}\right)$ are always above this region towards the center. For large magnetic diffusion $\left(\eta_{m}=6 \times 10^{27} \mathrm{~cm}^{2} \mathrm{~s}^{-1}\right)$ half of the simulated profiles are above the best fit model, the other half below the best fit model in the central part. From that, we conclude that a value of $\eta_{m}=6 \times 10^{27} \mathrm{~cm}^{2} \mathrm{~s}^{-1}$ (20 in the code internal units) is the one that provides the best match with that inferred from Coma cluster observations. The numerical diffusion inside the SPH smoothing length is of the order of $10^{18} \mathrm{~cm}^{2} \mathrm{~s}^{-1}$ that is several orders of magnitudes lower than the one we have implemented as magnetic resistivity, and thus does not affect our results.

\subsection{Physical origin of the magnetic resistivity}

In the previous Sections we have shown that a relatively large value of $\eta_{m}$ is required in the induction equation (Eq. 2) to match the radial profile and the central value of the magnetic field inferred from 

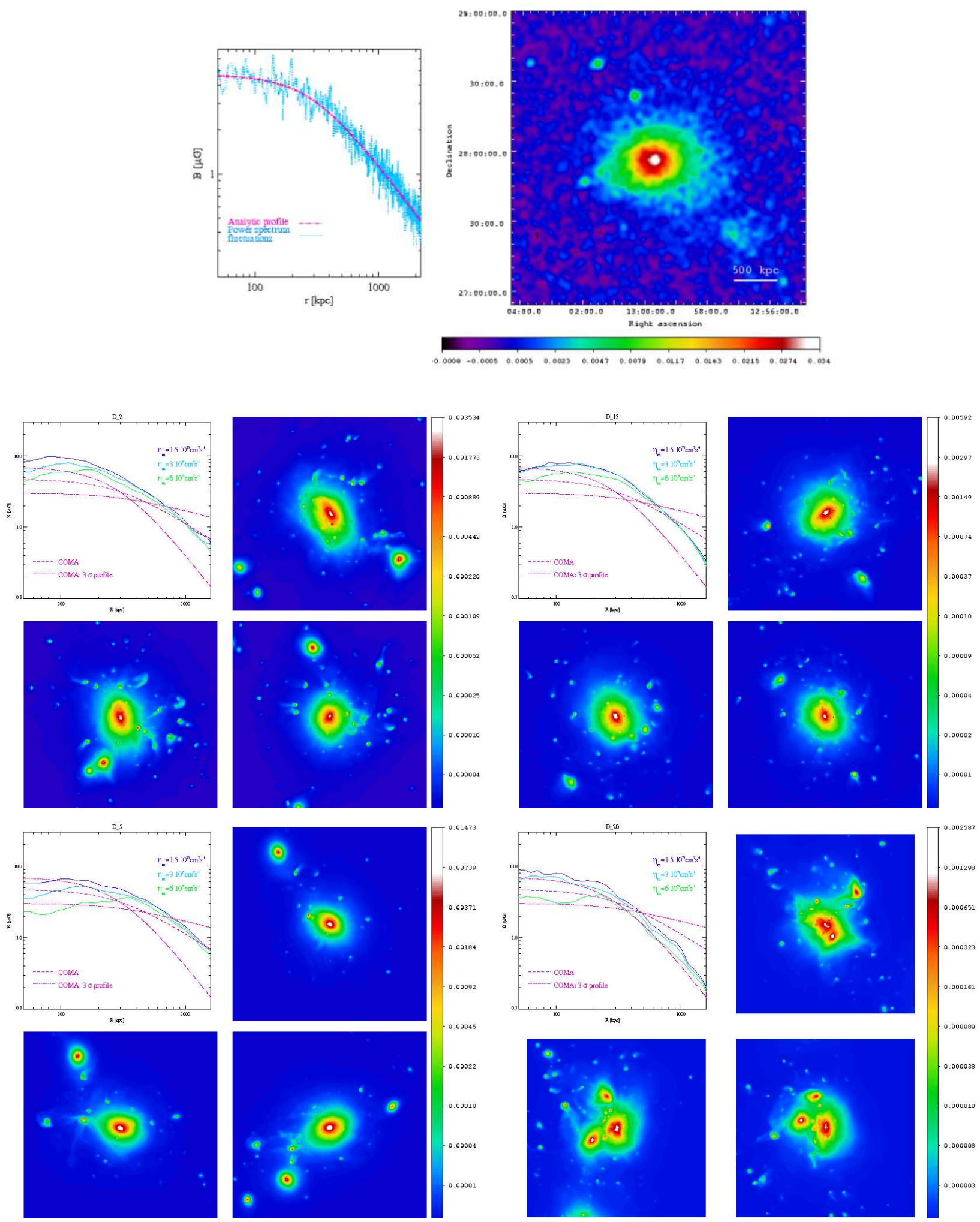

Figure 4. Top: The right panel shows the Coma cluster X-ray surface brightness from the ROSAT All Sky Survey, in the energy band 0.1- 2.4 keV (color coded). The shown region size is $\sim 3 \times 3 \mathrm{Mpc}$, corresponding to $\sim 1 R_{\mathrm{vir}} \times 1 R_{\mathrm{vir}}$. The left panel shows the magnetic field profile that gives the best fit to Faraday rotation observations (see Bonafede et al. 2010 for details). Bottom: Projected X-ray surface brightness for the clusters used to test the value of $\eta_{m}$. (Top: D_2 (left), D_13 (right); Bottom: D_5 (left), D_20 (right))x. X Y and Z projection are shown in the upper right, lower left and right sub-panels of each panel. The size of the projected X-ray images corresponds to $2 R_{\mathrm{vir}}$. The magnetic field radial profile $(\langle B\rangle)$ is plotted in the upper left panel for values of $\eta_{m}=1.3,3$ and $6 \times 10^{27} \mathrm{~cm}^{2} \mathrm{~s}^{-1}$ (5, 10 and 20 in code units). Magenta lines indicate the profile of the Coma cluster as inferred from radio RM observations. The shaded line represents the best fit, the dotted-shaded lines indicate the flatter and shallower profile that are compatible within $3 \sigma$ with radio RM data (see Bonafede et al. 2010 for further details). 
Coma cluster observations. In order to correctly interpret this result, it must be kept in mind that the induction equation (Eq.2) describes the evolution of a magnetic field $B$ at our resolution limit (which is of order of $10 \mathrm{kpc}$ ). The turbulent cascade is expected to develop down to smaller scales, where unresolved turbulent motions would contribute to the diffusion described by $\eta_{m}$. Hence, we can define the diffusion coefficient $\eta_{m}$ as

$\eta_{m}=\eta_{\text {Coulomb }}+\eta_{\text {turb }}$,

with $\eta_{\text {Coulomb }}$ related to the thermal conductivity $\sigma$ by

$\eta_{\text {Coulomb }}=\frac{c^{2}}{4 \pi \sigma}$.

Following Spitzer (1956), when the mean free path is determined by Coulomb collisions, the thermal conductivity of the plasma can be expressed as

$\frac{1}{\sigma}=\frac{\pi e^{2} m_{e}^{1 / 2}}{\left(4 \pi \epsilon_{0}\right)^{2}(k T)^{3 / 2}} \ln (\Lambda)$,

$\Lambda$ being the Coulomb logarithm. For a typical cluster environment (e.g.densities $n \approx 10^{-2} \mathrm{~cm}^{-3}$ and temperatures $T \approx 10^{80} \mathrm{~K}$ ), the diffusion coefficient is:

$\eta_{\text {culomb }} \approx 2 \times 10^{13} T^{-3 / 2} \approx 20 \mathrm{~cm}^{2} \mathrm{~s}^{-1}$.

Hence, the diffusion coefficient, arising from the gas thermal conductivity, does not significantly contribute to the evolution of $B$ in the induction equation. On the other hand, in a turbulent plasma the motion of charges will be a random walk characterized by a length scale $\lambda_{\text {turb }}$ and by a velocity $v_{\text {turb }}$. Following Dennis \& Chandran (2005), the plasma turbulent diffusion coefficient $\eta_{\text {turb }}$ can be defined as:

$\eta_{\text {turb }} \sim 0.1 \lambda_{\text {turb }} \times v_{\text {turb }}$

Typical values of $v_{\text {turb }}$ at our resolution of several kpc, corresponding to scales $\lambda_{\text {turb }}$ that fall below our resolution limit, will be several tens of $\mathrm{km} \mathrm{s}^{-1}$, and will lead to diffusion coefficients similar to the one that we used in our simulations (see Table 2). Estimates of $v_{\text {turb }}$ at these small spatial scales cannot be provided by any observation so far. However, it is possible to infer such estimates from the values of $v$ obtained at larger spatial scales, assuming that the turbulent power spectrum can be described by a single power-law down to the small scales of interest. Using X-ray data, Schuecker et al. (2004) derived pseudo-pressure fluctuations maps of the gas in the Coma cluster. They revealed the presence of a scale-invariant pressure fluctuation spectrum, that is consistent with the Kolmogorov slope, and could estimate the size of the turbulent eddies in the range from $40 \mathrm{kpc}$ to $100 \mathrm{kpc}$. On smaller scales, the number of photons detected were not sufficient for a reliable pressure measurement. The energy content associated with these turbulent motions is estimated to be roughly $10 \%$ of the thermal one (Schuecker et al. 2004). The sound velocity within the Coma cluster $\left(T \sim 10^{8} \mathrm{~K}\right)$ is $\sim 1500 \mathrm{~km}^{-1}$. Therefore the turbulent velocities associated with the largest scales $(\approx 100 \mathrm{kpc})$ found by Schuecker et al. (2004) would correspond to $\sim 470 \mathrm{~km} \mathrm{~s}^{-1}$. Assuming a Kolmogorov-like power spectrum, this translates into a turbulent velocity of $\sim 30 \mathrm{kms}^{-1}$ at a length scale of $2 \mathrm{kpc}$, that is the minimum scale revealed by Rotation Measure observations (Bonafede et al. 2010). A turbulent velocity of $\sim 30 \mathrm{kms}^{-1}$ at 2 kpc would yield to $\eta_{m} \sim 2 \times 10^{27} \mathrm{~cm}^{2} s^{-1}$, similar to the value we have used in the simulations. A sample of clusters for which $v_{t u r b}$, $\lambda_{t u r b}$, and the power spectrum slope are estimated observationally would of course allow us a better and more reliable comparison.
Although such observations are not available in the literature so far, another estimate for $\eta_{\text {turb }}$ has been derived by Rebusco et al. (2006). The authors have analyzed the effect of turbulent diffusion on the iron abundance profiles in the ICM for a sample of clusters, finding $\eta_{\text {turb }}$ in the range $6 \times 10^{27}-4.5 \times 10^{29}$.

Estimates of $v_{\text {turb }}$ and $\lambda_{\text {turb }}$ can also be derived by cosmological simulation. Different numerical schemes (e.g. Dolag et al. 2005; Vazza et al. 2006; Iapichino \& Niemeyer 2008; Vazza et al. 2009) have been optimized to follow the evolution of turbulent flows within the ICM of simulated galaxy clusters (see also Zhuravleva 2011). These works indicate that the energy in turbulent motions is $\sim 10-20 \%$ of the thermal one at $z=0$ within the virial radius. In particular, Maier et al. (2009) have measured the spectral properties of the gas velocity field, finding a good agreement with the Kolmogorov power spectrum slope over scales ranging from 300 kpc down to the scale correspondent to the Nyquist frequency. The velocity of the turbulent eddies at scales of $10 \mathrm{kpc}$ is estimated to be $\sim 50-100 \mathrm{~km} \mathrm{~s}^{-1}$, resulting is $\eta_{m} \sim \times 10^{28}$, in good agreement with the values adopted in the simulations presented here. In addition, Maier et al. (2009) have found $\eta_{\text {turb }} \sim 2 \times 10^{28} \mathrm{~cm}^{2} \mathrm{~s}^{-1}$, using $\lambda_{\text {turb }}=7.8 \mathrm{kpc} \mathrm{h}^{-1}$ and $v_{\text {turb }}=60 \mathrm{kms}^{-1}$. It is also worth mentioning that the value of the diffusion coefficient $\eta_{m}$ is within the range $\eta_{m} \approx 3 \times 10^{25} \mathrm{~cm}^{2} \mathrm{~s}^{-1}-\eta_{m} \approx 3 \times 10^{29} \mathrm{~cm}^{2} \mathrm{~s}^{-1}$ which are the values needed to operate a cosmic ray driven dynamo within a galaxy (see Siejkowski et al.2010) and to power the Coma radio halo by an in-situ acceleration model respectively ${ }^{2}$ (see Schlickeiser et al. 1987).

All these different values for $\eta_{m}$ are reported in Table 3 In summary we can conclude that our inferred value of $\eta_{m} \approx 6 \times$ $10^{27} \mathrm{~cm}^{2} \mathrm{~s}^{-1}$ at our unresolved scales of $\sim 10 \mathrm{kpc}$ is well in range with what would be expected from turbulent motions within the ICM.

\subsubsection{About the use of a constant $\eta_{m}$}

The Equations 11 and 15 clarify the physical origin of the resistivity term that we have implemented in the induction equation. In this work, the value of $\eta_{m}$ has been kept constant throughout the whole re-simulations. It is clear that having a $\eta_{m}$ that changes as a function of $v_{t u r b}$ and $\lambda_{t u r b}$ locally would allow one to follow the evolution of the magnetic field more properly. Identifying the turbulent motions to compute the most correct value of $\eta_{m}$ at every step during the simulation is however not feasible. Different algorithms have been developed to identify and analyze the turbulent motions (see e.g. Dolag et al. 2005; Maier et al. 2009; Vazza et al. 2011). These algorithms need to subtract large-scale laminar motions before revealing the turbulent patterns, and can then be applied in the post processing once the simulation is run. We can verify the validity of the assumed constant value of $\eta_{m}$ by checking which values of $\lambda_{t u r b}$ and $v_{t u r b}$ are obtained at different distances from the cluster center by the above mentioned works. (Maier et al. 2009) have computed the profile of the turbulent velocity for a simulated galaxy cluster. The velocity profile, once scaled at the length scale of $7.8 \mathrm{kpc}$ - the highest resolved region of the simulation- shows a rather flat profile, with values ranging from $\sim 50$ to $\sim 90 \mathrm{Kms}^{-1}$ within the cluster virial radius. This implies that the assumption of

2 We note however that the re-acceleration model proposed in that work is due to Alfen modes, while more recent works indicate that the re-acceleration is due to MHD modes at very small scales, see e.g. Brunetti \& Lazarian (2011). 


\begin{tabular}{|c|c|c|c|c|}
\hline Cluster name & $\begin{array}{l}B_{0} \\
\mu \mathrm{G}\end{array}$ & $\begin{array}{c}r_{c} \\
\mathrm{kpc}\end{array}$ & $\mu$ & $\chi^{2}$ \\
\hline D_1 & 4.7 & 339 & 0.40 & 0.9 \\
\hline D_2 & 6.8 & 295 & 0.52 & 0.7 \\
\hline D_3 & 2.5 & 361 & 0.33 & 1.8 \\
\hline D_4 & 2.7 & 285 & 0.43 & 1.2 \\
\hline D_5 & 3.0 & 346 & 0.35 & 1.7 \\
\hline D_6 & 5.1 & 414 & 0.57 & 0.9 \\
\hline D_7 & 6.5 & 362 & 0.58 & 1.1 \\
\hline D_8 & 3.5 & 342 & 0.34 & 1.0 \\
\hline D_9 & 5.4 & 404 & 0.49 & 0.9 \\
\hline D_10 & 3.9 & 319 & 0.38 & 1.9 \\
\hline D_11 & 3.9 & 293 & 0.31 & 2.0 \\
\hline D_12 & 3.3 & 415 & 0.51 & 1.4 \\
\hline D_13 & 6.5 & 332 & 0.59 & 1.0 \\
\hline D_14 & 4.6 & 329 & 0.44 & 0.7 \\
\hline D_15 & 2.9 & 344 & 0.30 & 1.9 \\
\hline D_16 & 5.6 & 354 & 0.54 & 0.9 \\
\hline D_17 & 5.5 & 431 & 0.43 & 1.1 \\
\hline D_18 & 6.4 & 327 & 0.63 & 1.1 \\
\hline D_19 & 2.6 & 341 & 0.29 & 1.7 \\
\hline D_20 & 4.3 & 323 & 0.63 & 0.7 \\
\hline D_21 & 2.8 & 311 & 0.43 & 1.9 \\
\hline D_22 & 4.6 & 423 & 0.46 & 0.9 \\
\hline D_23 & 9.9 & 241 & 0.53 & 1.2 \\
\hline D_24 & 4.9 & 375 & 0.55 & 1.0 \\
\hline Mean values & $4.7 \pm 1.7$ & $346 \pm 47$ & $0.46 \pm 0.11$ & - \\
\hline Coma Cluster & $4.7_{-0.8}^{+0.7}$ & $291 \pm 17$ & $0.38_{-0.09}^{+0.17}$ & - \\
\hline
\end{tabular}

Table 4. Results of the fit of the magnetic field profiles to Eq.16

a constant $\eta_{m}$, although not optimal, is well justified in our case. The simulations presented here represent a good starting point to investigate for the first time the effects related to such resistivity term.

\subsection{Magnetic properties of massive clusters}

We finally simulated the whole cluster sample, fixing the magnetic resistivity to our inferred value of $\eta_{m}=6 \times 10^{27} \mathrm{~cm}^{2} \mathrm{~s}^{-1}$. Therefore, we can study, for the first time, the scatter of the magnetic field properties in massive clusters using a volume limited sample. Figure 5 shows the mean magnetic field within $0.3 \times R_{\text {vir }}$, corresponding to roughly $1 \mathrm{Mpc}$ for our set of massive clusters. The simulations scatter mildly around the value inferred from observations of Coma. Our mean value is $2.6 \mu \mathrm{G}$ with and $\mathrm{rms}$ of $0.6 \mu \mathrm{G}$. We point out here again that the sample of massive clusters comprises objects that have very different dynamical state at $z=0$. It is interesting to note that the mean magnetic field, averaged over the central $\mathrm{Mpc}^{3}$, does not depend on the present dynamical state of the cluster at $z=0$ (see also Section 5)

\subsubsection{Magnetic field profiles of massive galaxy clusters}

In Figure 6the magnetic field profiles are shown for all the clusters in the sample. In the right panel of that Figure the mean and the dispersion of the magnetic field profiles are compared with the best fit for the Coma cluster. It is worth stressing that the exact shape of the profile inferred from Coma observations is given ad hoc as a parametric model to fit the data. Hence, it is not clear how significant the differences between simulations and observations in the exact shape are. Nonetheless, the fit to the observations lies completely within the scatter of the profiles predicted by our simulations. This is a non-trivial result, confirming previous findings that the magnetic field in galaxy clusters is shaped by the (turbulent) motions within the ICM and therefore reflects a natural prediction of the structure formation process.

Although the mean magnetic field profile shows a good agreement with the one inferred from Coma observations, there are differences in the shape of the individual profiles, likely reflecting the dynamical state and the different morphologies of the individual objects. Magnetic field profiles are usually compared to the gas density profiles, in order to derive a scaling with the radial distance from the cluster center. Here we adopt another approach and fit the magnetic field profiles directly to a " $\beta$ model-like" profile (Cavaliere \& Fusco-Femiano 1976), that for magnetic fields is usually written as:

$B(r)=B_{0}\left(1+\frac{r^{2}}{r_{c}^{\prime 2}}\right)^{-\frac{3}{2} \mu}$

where $B_{0}, r_{c}$, and $\mu$ are free parameters. The fits have been performed in the range $50-2000 \mathrm{kpc}$, to properly compare with the results obtained from Coma observations. The results of the fit are shown in Table 4 The mean values of $B_{0}$ and $\mu$ are reported is the last row of the Table and compared to those of the Coma cluster. While previously numerical simulations indicated a steeper profile of the magnetic field with the gas density (i.e. $\alpha \sim 1$ in Eq. 10], and thus with the radial distance from the cluster center, now the effect of the magnetic resistivity is that of flattening the profiles, reaching a better agreement with observations. In the case of the Coma cluster, a value of $\alpha=0.5_{-0.1}^{+0.2}$ gives the best fit with the observations, corresponding to $\mu \sim 0.38_{-0.09}^{+0.17}$, in very good agreement with the mean of the best fit for our simulated clusters, that is $\mu \sim 0.46 \pm 0.11$. Hence, not only the mean value of $B$ over the central $\mathrm{Mpc}^{3}$ has a small dispersion in this high-mass cluster set, but also the central value of $B_{0}$, and its slope with the gas density, as derived from the beta-model fit, are quite similar.

\subsection{Magnetic field and thermal properties}

We present in this Section a first overview of the thermal properties of the ICM in the presence of a diffusive magnetic field. A more detailed analysis will be performed in a second paper, where the whole sample will be analyzed. Here we investigate if and how the presence of resistive magnetic field may affect the ICM properties of the four clusters we have simulated with different values of $\eta_{m}$. In Figure 7 the density, temperature, and entropy profiles of these clusters are shown for different values of $\eta_{m}$. The profiles converge at distances larger than few $\%$ of the virial radius, while differ in the very inner region of the clusters. As mentioned in Section 2.3 the magnetic field energy that is dissipated during the cluster formation is transformed into heat. Although the dynamical effect of a magnetic field of the order of $\sim 1-10 \mu \mathrm{G}$ in the cluster cores is negligible, the overall effect of the magnetic force and pressure integrated over a Hubble time results in a change of the density and temperature profile. As the resistivity constant $\eta_{m}$ increases, the amount of magnetic field energy, that is dissipated and hence converted into heat, increases accordingly. An additional source of heating is then present in the cluster central region, that has the effect of flatten- 

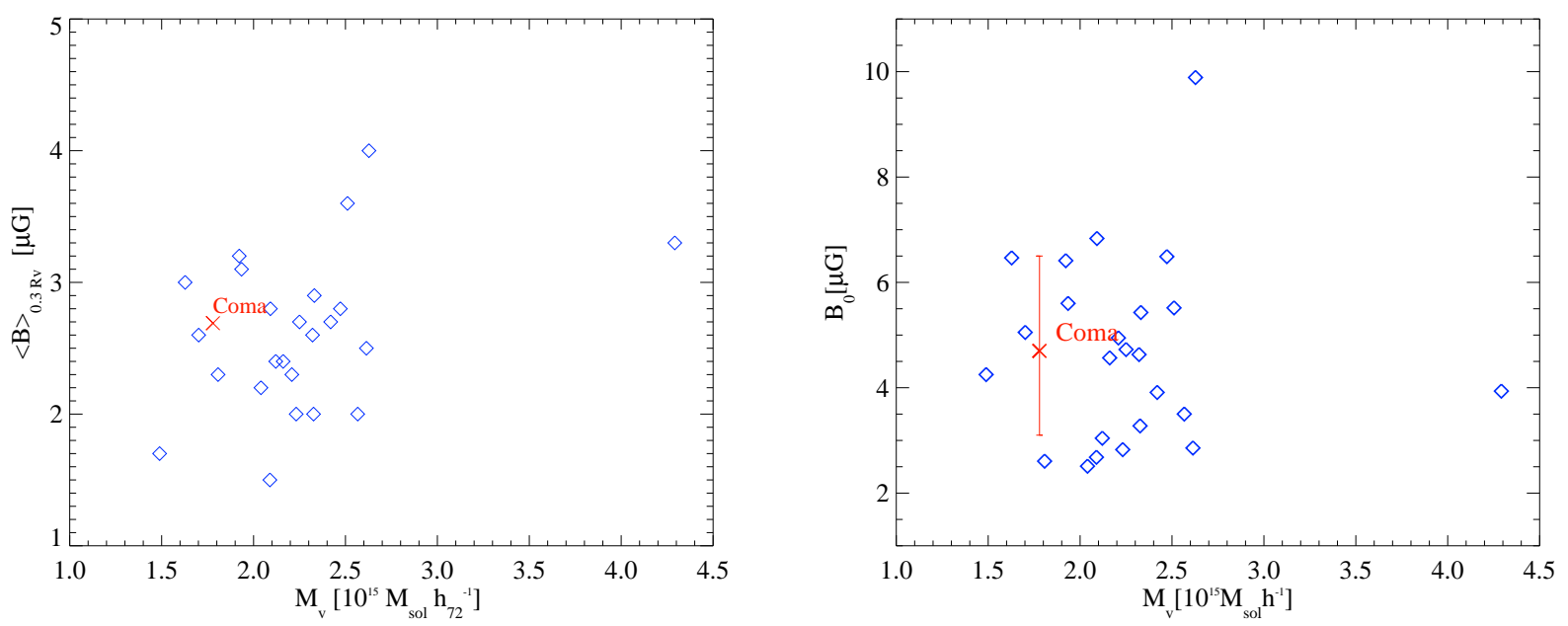

Figure 5. Left: Magnetic field averaged over the central $0.3 R_{\mathrm{vir}}$ versus virial mass of the our cluster set (Blue diamonds). The red cross refers to the mean magnetic field for the Coma cluster. The error-bar refers to the $3 \sigma$ of the $c h i^{2}$ given by Bonafede et al. 2010). Right: Magnetic field in the cluster center, as results from the fit of a $\beta$-model profile, versus the cluster virial mass.
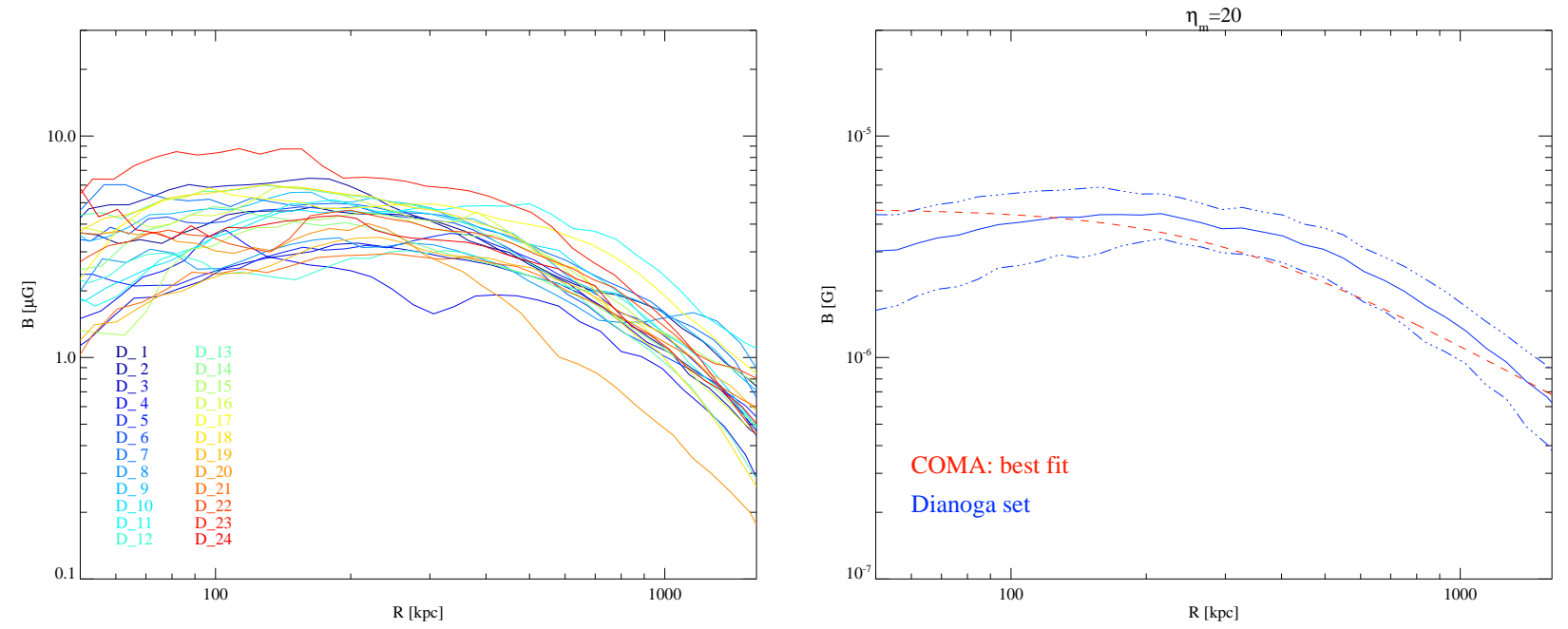

Figure 6. Left: magnetic field strength profile for the whole cluster set. Right: in blue mean (continuous line) and dispersion (dot-dashed) of the magnetic field strength profile. Red dashed line refers to the best fit obtained from RM observations for the Coma cluster (Bonafede et al. 2010).

ing the temperature profile. The higher pressure that would result from a higher temperature is then balanced by reducing the gas density in the cluster central region, up to a factor 2 . The temperature and density profiles do not change adiabatically, as demonstrated by the entropy profiles of the clusters. The entropy, computed as $S=k T / n^{2 / 3}$, flattens in the inner region of the clusters, indicating that the transport of low entropy gas in inhibited.

In Figure 8 the magnetic field within $0.3 \times R_{\mathrm{vir}}$, is plotted versus the cluster mean temperature computed over the same region. Although the sample is small, and the value of the magnetic fields within $0.3 \times R_{\text {vir }}$ varies of a factor $\sim 2$, a trend is suggested. Magnetic field in higher temperature clusters seem to be higher. The correlation should be better investigated with a higher sample of simulated galaxy clusters, since observational data do not suggest a trend of the $R M$ in clusters depending on the temperature (Govoni et al. 2010). We note also that such a trend is much less visible when the value of $B_{0}$, resulting from the $\beta$-model fit is compared with the cluster mean temperature (Figure 8 right panel).

\section{DISCUSSION AND CONCLUSIONS}

We have presented a set of simulated galaxy clusters. It consists of 24 massive objects $\left(M_{\mathrm{vir}}>10^{15} h^{-1} M_{\odot}\right)$ re-simulated at high resolution up to 5-6 virial radii, plus 50 more clusters with $M_{\text {vir }}>10^{14} h^{-1} M_{\odot}$ that fell within this high resolution region. This large set permits to study the cluster properties in a wide range of masses and at high resolution (see e.g. Fabjan et al. 2011). The evolution of the clusters has been followed using the MHD implementation within the GADGET - 3 code (Dolag \& Stasyszyn 2009), that has been here modified in order to include the magnetic resistivity term in the induction equations. It is the first time that this 

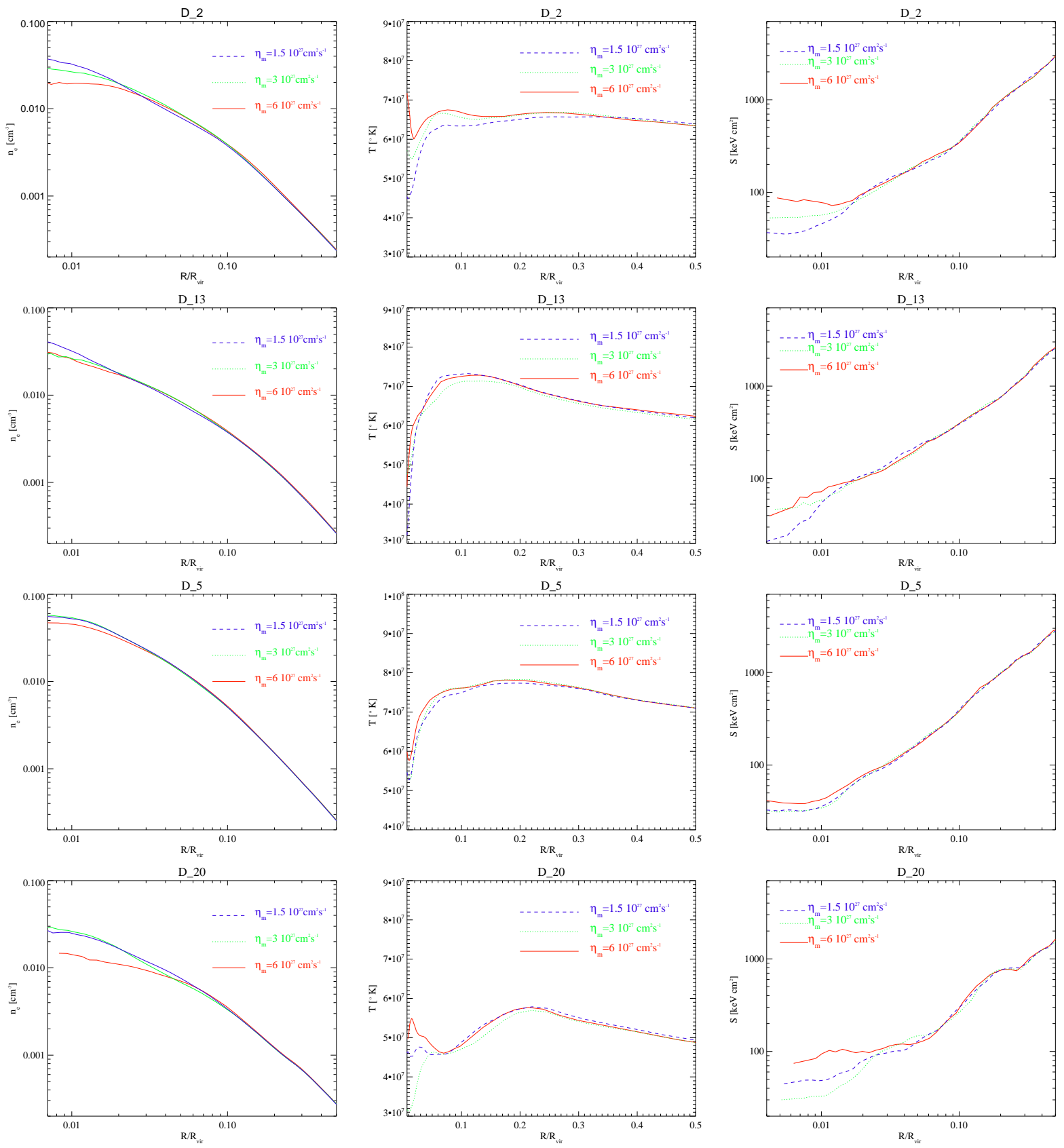

Figure 7. Density (left column), temperature (middle column) and entropy (right column) profiles for the clusters D_2, D_5, D_13 and D_20 from top to bottom respectively. Different colors refer to different values of the magnetic resistivity constant $\eta_{m}$, as indicated in the panels.

term is analyzed in the context of cluster formation and evolution. In this first paper we have presented the zoomed initial conditions, the non-ideal MHD implementation, and the average properties of the more massive clusters when a magnetic resistivity term is included in the MHD equations. Further analysis will be performed in a future paper (Paper II, Bonafede et al. in prep) where the physical implications will be discussed in more detail.

Our main results can be summarized as follows:

- Non-ideal MHD equations have been implemented within the GADGET code. The tests performed on two different problems show that the numerical implementation is accurate and can be used to study the effect of the magnetic resitivity.
- The magnetic field profiles obtained with non-ideal MHD can reproduce the profile inferred from Faraday Rotation Measures of the Coma cluster. Four clusters having X-ray morphologies similar to the one of the Coma cluster have been selected to test the effect of changing the constant $\eta_{m}$ used in the induction equation. The best agreement with the limits given by observations is achieved with $\eta_{m}=6 \times 10^{27} \mathrm{~cm}^{2} \mathrm{~s}^{-1}$.

- The whole sample has been simulated using $\eta_{m}=6 \times$ $10^{27} \mathrm{~cm}^{2} \mathrm{~s}^{-1}$, and the derived magnetic field profiles are consistent with the Coma profile. The best-fit found for the Coma profile lies in fact between the rms of the simulated profiles. 

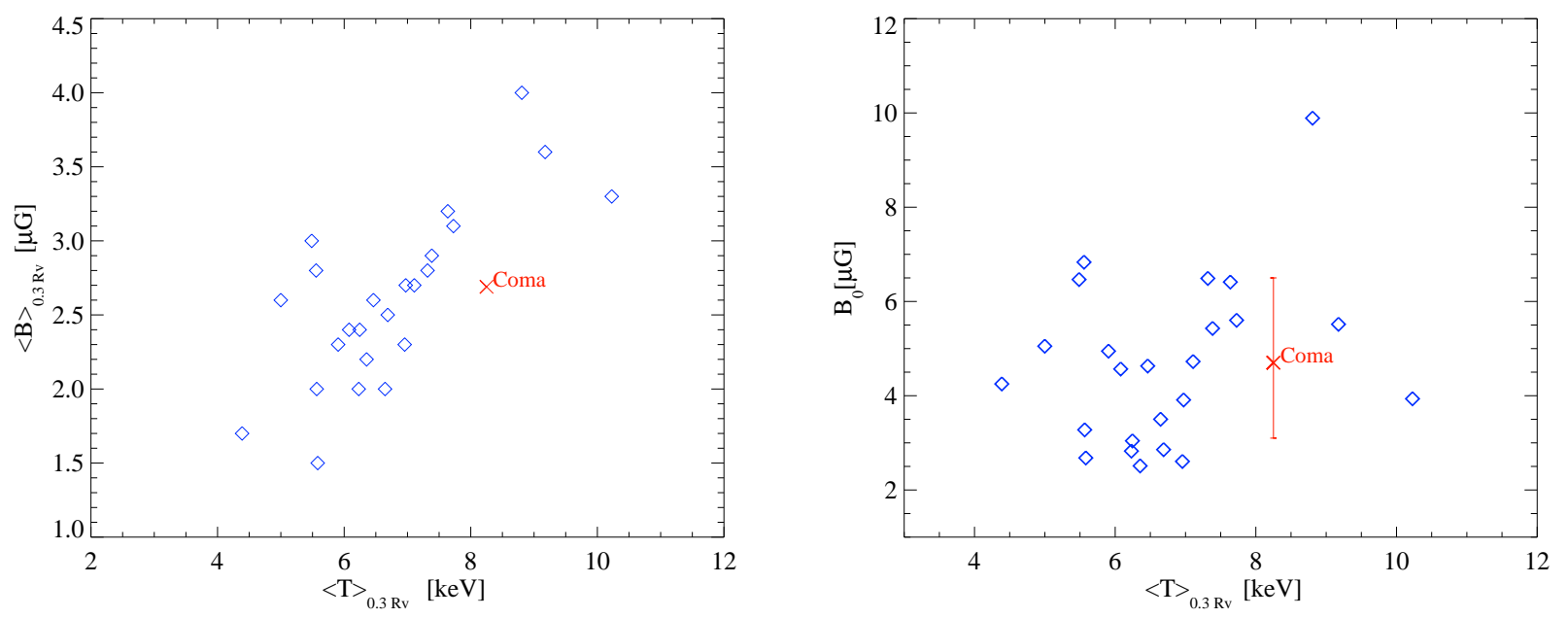

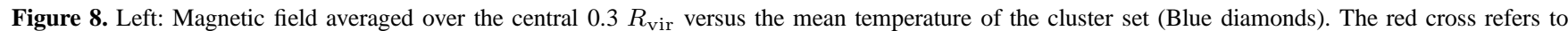
the mean magnetic field for the Coma cluster. The error-bar refers to the $3 \sigma$ of the $c h i^{2}$ given by Bonafede et al. (2010). Right: Magnetic field in the cluster center, as results from the fit of a $\beta$-model profile versus the cluster mean temperature inside $0.3 R_{\mathrm{vir}}$. The temperature for the Coma cluster is the one given by Arnaud et al. (2001), computed inside 0.25 of the Coma virial radius. All the quantities are computed from the dissipative simulation runs with $\eta_{m}=6 \times 10^{27} \mathrm{~cm}^{2} \mathrm{~s}^{-1}$.

- We have fitted the magnetic field profile with a $\beta$-like model, finding that the magnetic field profile of the simulated galaxy clusters can be well reproduced by values $B_{0}=4.7 \pm 1.7$, and $\mu=0.46 \pm 0.11$ (see Eq. 16, in good agreement with the value found for the Coma cluster. The value of $\mu$ would correspond to a value of $\alpha \sim 0.6$ (Eq. 10) for a Coma-like gas density profile.

- We have investigated possible correlations of the magnetic field strength with the cluster mass. The magnetic field strength, averaged over a central spherical volume of $0.3 R_{\mathrm{vir}} h^{-1}$ in radius, is similar for all the clusters in the sample, in agreement with what has been recently found by Bonafede et al. (2011). This indicates that the presence of radio halo emission, found in a fraction of massive galaxy clusters, cannot be attributed to a difference in the magnetic field strength. A mild dependence of the magnetic field strength with cluster temperature is indicated by these simulations.

- The density, temperature and entropy profiles of the simulated galaxy clusters have been derived for different values of $\eta_{m}$. We find that the effect of a magnetic diffusive constant is visible in such profiles, leading to flatter temperature and entropy profiles in the inner region of the cluster ( $R \leqslant 0.1 R_{\mathrm{vir}}$ at maximum).

The cluster sample and the new MHD-implementation we have presented is suitable to investigate other issues that are not discussed here, and that will be studied in a future paper, such as the interplay of the magnetic field with the thermal gas of the ICM (e.g.how is the thermal conduction modified, the role of the magnetic pressure in suppressing the cooling in the inner regions). In the next years, the LOw Frequency ARray (LOFAR) and the Expanded Very Large Array (EVLA) will allow us to improve our knowledge of the nonthermal component of the ICM, and a larger sample of data will be soon available for a more complete comparison.

\section{ACKNOWLEDGMENTS}

We thank R. Brunino and C. Gheller for their support at CINECA Supercomputing Center, G. Tormen for providing the ZIC code, and M. Brüggen and F. Vazza for the useful discussions and comments. We thank the referee for his/her comments that helped is clarifying the paper in several parts. A.B. thanks the MPA in Garching for the hospitality and the support by the Marco Polo exchange program of the Bologna University. We acknowledge the use of computational resources under the CINECA-INAF 20082010 agreement. AB and FS acknowledge support by the DFG Research Unit 1254 "Magnetization of interstellar and intergalactic media: the prospect of low frequency radio observations". K.D. acknowledges the support by the DFG Priority Program 1177 and additional support by the DFG Cluster of Excellence "Origin and Structure of the Universe".

\section{REFERENCES}

Arnaud M., Aghanim N., Gastaud R., Neumann D. M., Lumb D., Briel U., Altieri B., Ghizzardi S., Mittaz J., Sasseen T. P., Vestrand W. T., 2001, A\&A, 365, L67

Bertschinger E., 1995, ArXiv Astrophysics e-prints

Böhringer H., Schuecker P., Pratt G. W., Arnaud M., Ponman T. J., Croston J. H., Borgani S., Bower R. G., Briel U. G., Collins C. A., 2007, A\&A, 469, 363

Bonafede A., Feretti L., Murgia M., Govoni F., Giovannini G., Dallacasa D., Dolag K., Taylor G. B., 2010, A\&A, 513, A30

Bonafede A., Govoni F., Feretti L., Murgia M., Giovannini G., Brüggen M., 2011, A\&A, 530, A24+

Borgani S., Kravtsov A., 2009, ArXiv e-prints

Børve S., Omang M., Trulsen J., 2001, ApJ, 561, 82

Brüggen M., Ruszkowski M., Simionescu A., Hoeft M., Dalla Vecchia C., 2005, ApJ, 631, L21

Brunetti G., Lazarian A., 2011, MNRAS, 410, 127

Buote D. A., 2001, ApJ, 553, L15

Cassano R., Ettori S., Giacintucci S., Brunetti G., Markevitch M., Venturi T., Gitti M., 2010, ApJ, 721, L82

Cavaliere A., Fusco-Femiano R., 1976, A\&A, 49, 137 
Clarke T. E., 2004, Journal of Korean Astronomical Society, 37, 337

Collins D. C., Xu H., Norman M. L., Li H., Li S., 2010, ApJS, 186,308

Davis M., Efstathiou G., Frenk C. S., White S. D. M., 1985, ApJ, 292, 371

Dennis T. J., Chandran B. D. G., 2005, ApJ, 622, 205

Dolag K., Bartelmann M., Lesch H., 1999, A\&A, 348, 351

Dolag K., Bartelmann M., Lesch H., 2002, A\&A, 387, 383

Dolag K., Bykov A. M., Diaferio A., 2008, Space Science Reviews, 134, 311

Dolag K., Grasso D., Springel V., Tkachev I., 2005, Journal of Cosmology and Astro-Particle Physics, 1, 9

Dolag K., Grasso D., Springel V., Trachev I., 2005, in L. O. Sjouwerman \& K. K. Dyer ed., X-Ray and Radio Connections Simulating the Magnetic Field in the Local Supercluster

Dolag K., Schindler S., Govoni F., Feretti L., 2001, A\&A, 378, 777

Dolag K., Stasyszyn F., 2009, MNRAS, 398, 1678

Dolag K., Vazza F., Brunetti G., Tormen G., 2005, MNRAS, 364, 753

Donnert J., Dolag K., Lesch H., Müller E., 2009, MNRAS, 392, 1008

Dubois Y., Teyssier R., 2008, A\&A, 482, L13

Eke V. R., Cole S., Frenk C. S., 1996, MNRAS, 282, 263

Fabjan D., Borgani S., Rasia E., Bonafede A., Dolag K., Murante G., Tornatore L., 2011, ArXiv e-prints

Ferrari C., Govoni F., Schindler S., Bykov A. M., Rephaeli Y., 2008, Space Science Reviews, 134, 93

Giovannini G., Bonafede A., Feretti L., Govoni F., Murgia M., Ferrari F., Monti G., 2009, A\&A, 507, 1257

Górski K. M., Hivon E., Banday A. J., Wandelt B. D., Hansen F. K., Reinecke M., Bartelmann M., 2005, ApJ, 622, 759

Govoni F., Dolag K., Murgia M., Feretti L., Schindler S., Giovannini G., Boschin W., Vacca V., Bonafede A., 2010, A\&A, 522, A105+

Govoni F., Enßlin T. A., Feretti L., Giovannini G., 2001, A\&A, 369,441

Govoni F., Murgia M., Feretti L., Giovannini G., Dolag K., Taylor G. B., 2006, A\&A, 460, 425

Iapichino L., Niemeyer J. C., 2008, MNRAS, 388, 1089

Jenkins A., 2010, MNRAS, 403, 1859

Johnston-Hollitt M., Hollitt C. P., Ekers R. D., 2004, in B. Uyaniker, W. Reich, \& R. Wielebinski ed., The Magnetized Interstellar Medium Statistical Analysis of Extra-galactic Rotation Measures. pp 13-18

Kotarba H., Karl S. J., Naab T., Johansson P. H., Dolag K., Lesch H., Stasyszyn F. A., 2010, ApJ, 716, 1438

Kotarba H., Lesch H., Dolag K., Naab T., Johansson P. H., Stasyszyn F. A., 2009, MNRAS, 397, 733

Laing R. A., Bridle A. H., Parma P., Murgia M., 2008, MNRAS, 391,521

Lesch H., Hanasz M., 2003, A\&A, 401, 809

Maier A., Iapichino L., Schmidt W., Niemeyer J. C., 2009, ApJ, 707, 40

Murgia M., Govoni F., Feretti L., Giovannini G., Dallacasa D., Fanti R., Taylor G. B., Dolag K., 2004, A\&A, 424, 429

Price D. J., Monaghan J. J., 2004a, MNRAS, 348, 123

Price D. J., Monaghan J. J., 2004b, MNRAS, 348, 139

Price D. J., Monaghan J. J., 2005, MNRAS, 364, 384

Price D. J., Monaghan J. J., 2007, MNRAS, 374, 1347
Rebusco P., Churazov E., Böhringer H., Forman W., 2006, MNRAS, 372, 1840

Schlickeiser R., Sievers A., Thiemann H., 1987, A\&A, 182, 21

Schuecker P., Finoguenov A., Miniati F., Böhringer H., Briel U. G., 2004, A\&A, 426, 387

Siejkowski H., Soida M., Otmianowska-Mazur K., Hanasz M., Bomans D. J., 2010, A\&A, 510, A97+

Spitzer L., 1956, Physics of Fully Ionized Gases

Springel V., 2005, MNRAS, 364, 1105

Springel V., Hernquist L., 2002, MNRAS, 333, 649

Springel V., Yoshida N., White S. D. M., 2001, New Astr, 6, 79

Strong A. W., Moskalenko I. V., Ptuskin V. S., 2007, Annual Review of Nuclear and Particle Science, 57, 285

Tormen G., Bouchet F. R., White S. D. M., 1997, MNRAS, 286, 865

Vazza F., Brunetti G., Gheller C., Brunino R., Brüggen M., 2011, A\&A, 529, A17+

Vazza F., Brunetti G., Kritsuk A., Wagner R., Gheller C., Norman M., 2009, A\&A, 504, 33

Vazza F., Tormen G., Cassano R., Brunetti G., Dolag K., 2006, MNRAS, 369, L14

Venturi T., Giacintucci S., Dallacasa D., Cassano R., Brunetti G., Bardelli S., Setti G., 2008, A\&A, 484, 327

Vogt C., Enßlin T. A., 2005, A\&A, 434, 67

White S. D. M., 1996, in R. Schaeffer, J. Silk, M. Spiro, \& J. ZinnJustin ed., Cosmology and Large Scale Structure Formation and Evolution of Galaxies. pp 349-+

Wilmot-Smith A. L., Priest E. R., Hornig G., 2005, Geophysical and Astrophysical Fluid Dynamics, 99, 177

Zhuravleva I., 2011, ArXiv e-prints

\section{APPENDIX A: GENERATING THE ZOOMED INITIAL CONDITIONS}

\section{A1 Dark matter re-simulations}

Creating zoomed initial conditions is essential to extend the dynamical range accessible through cosmological simulations, which is needed to study the detailed structure of objects, like e.g.galaxy clusters, with appropriate resolution. Since hydrodynamic simulations are sensitive to boundary conditions, regions around galaxy clusters have to be re-simulated with high resolution as well. In the last years the peripheral regions around galaxy clusters are also attracting more and more interest, given the increased sensitivity of modern instruments. Here we have optimized our initial conditions to study a statistical sample of massive clusters with reasonable computational resources. Our procedure is based on the ZIC code (Tormen et al. 1997) and we describe here the iterative procedure that we have used to obtain such highly optimized, zoomed initial conditions for our cluster sample.

We started from a large, cosmological, dark-matter only simulations, performed according to the 'concordance' $\Lambda$ CDM cosmological model $\left(\Omega_{\Lambda}=0.76, \Omega_{0}=0.24, h=0.72\right.$ and $\left.\sigma_{8}=0.8\right)$. The spectral index of power spectrum for the primordial density fluctuations $\left(P(k) \propto k^{n}\right)$ is $n=0.96$. This simulation, that we refer to as 'parent simulation', was carried out with the massively parallel TREE+SPH code GADGET-2 (Springel 2005) and consists of a periodic box of $1 h^{-1} \mathrm{Gpc}$ size. The cluster identification was performed at $z=0$ using a standard Friend of Friends algorithm. The linking length was fixed to 0.17 of the mean inter-particle separation between DM particles, to reflect the virial over-density for 
Table A1.

\begin{tabular}{|c|c|c|c|}
\hline Cluster & $\begin{array}{l}R \text { cleaned } \\
\quad\left[R_{\text {vir }}\right]\end{array}$ & $\begin{array}{c}M_{D M} \\
\left.\text { with } \mathrm{M}>10^{14}\right] h^{-1} M_{\odot}\end{array}$ & $\mathrm{N}$ of nearby clusters \\
\hline D_1 & 5.2 & 1.618 & 1 \\
\hline D_2 & 5.4 & 1.518 & 3 \\
\hline D_3 & 5.3 & 1.49 & 2 \\
\hline D_4 & 5.4 & 1.482 & \\
\hline D_5 & 5.0 & 1.537 & 4 \\
\hline D_6 & 5.0 & 1.165 & 4 \\
\hline D_7 & 5.4 & 1.776 & 1 \\
\hline D_8 & 5.3 & $1.993,1.170$ & 4 \\
\hline D_9 & 5.2 & 1.657 & \\
\hline D_10 & 5.1 & 1.705 & 6 \\
\hline D_11 & 5.3 & 3.163 & 1 \\
\hline D_12 & 5.5 & 1.678 & 2 \\
\hline D_13 & 5.6 & 1.171 & 4 \\
\hline D_14 & 6.0 & 1.557 & 3 \\
\hline D_15 & 5.5 & 1.840 & 1 \\
\hline D_16 & 5.2 & 1.385 & \\
\hline D_17 & 5.5 & 1.813 & \\
\hline D_18 & 5.1 & 1.356 & 1 \\
\hline D_19 & 5.1 & 1.316 & 1 \\
\hline D_20 & 5.2 & 1.067 & 2 \\
\hline D_21 & 5.9 & $1.623,1.011$ & 4 \\
\hline D_22 & 5.2 & 1.674 & \\
\hline D_23 & 5.1 & 1.880 & 3 \\
\hline D_24 & 5.0 & 1.507 & 3 \\
\hline
\end{tabular}

the adopted cosmology. Given the large volume this cosmological box contains a large sample of 64 clusters with $M_{F O F}>10^{15} h^{-1}$ $M_{\odot}$ at $z=0$. We selected the 24 most massive clusters for high resolution re-simulations. Figure A1 shows the projected density within $125 h^{-1} \mathrm{Mpc}$ slices of the parent simulation at $\mathrm{z}=0$. The positions of the 24 most massive clusters used in this work are marked by diamonds. From the final output of the DM only run, all of the particles out to a distance of $\approx 5-7 R_{\mathrm{vir}}$ around the cluster center were selected and then traced back to their initial positions. The corresponding Lagrangian region was enclosed in a box of side $L_{\mathrm{HR}} \sim 62.5 \mathrm{Mpc}$, the high resolution $(\mathrm{HR})$ region. Since the volume occupied by the HR particles, $V_{\mathrm{HR}}$, is usually only a fraction of the volume of the box $\left(L_{\mathrm{HR}}^{3}\right)$, we sampled the box with $64^{3}$ cells, and we marked cells which were actually occupied by the particles. In order to obtain a volume with a concave shape and no holes in it, some more cells were marked around/within $V_{\mathrm{HR}}$. The particles that occupy the marked cells were then traced back to the initial redshift of the simulation. The right panel of Figure A3 shows a cut through the $L_{\mathrm{HR}}$ volume. The blue cells trace the $V_{\mathrm{HR}}$ region, while the additional cells marked to obtain a concave volume are marked in red and green. This volume (defined as "occupied volume") was re-sampled with a higher number of particles in order to obtain a higher mass resolution (in this case of $1 \times 10^{9} h^{-1} M_{\odot}$ for DM particles). Particles were displayed according to a glass-like particle distribution (White 1996). The HR particles were perturbed using the same power spectrum of the parent simulation, keeping the same amplitudes and phases. New fluctuations at smaller spatial scales were added, since smaller frequencies are now sampled by the higher resolution particles. The amplitude of the fluctuations are given by the theoretical power spectrum $P(k)$ of the parent simulation, but extended to higher $k$. To minimize any changes in the tidal forces acting onto the high resolution region, we created a buffer region around the HR region, and sampled it with the same mass resolution as the parent cosmological simulation. The remaining volume of the simulation was re-sampled at lower resolution according to the following procedure: the density and velocity fields of the LR particles were re-sampled onto a spherical grid having constant angular resolution $d \theta$. The size of each cell $d r=r d \theta$ was chosen to obtain approximately cubic cells through the sphere. The interpolation onto a spherical grid reduces the number of LR particles to the minimum necessary to preserve the large-scale tidal field of the original simulation. We used $d \theta=1.5^{\circ}$, resulting in $\sim 2 \times 10^{6}$ low resolution particles, that guarantees an accurate sampling of the tidal field (see Tormen et al. 1997). By construction, as the distance from the HR region increases, $d r$ increases too, and the mass of the LR particles increases accordingly. The overall volume simulated for each cluster is the same as the parent simulation, ensuring that the forming structures correspond to the same that formed within the original cosmological simulation. The new initial conditions were finally traced back to a higher redshift $($ e.g. $z=70)$ to ensure that the $r m s$ of the particle displacement in the HR region is still small enough to guarantee the validity of the Zeldovich approximation. After generating the new IC at higher resolution, we re-run further dark matter-only re-simulation to verify that the volume of the HR region around each cluster was free from contamination of LR particles. Several iterations (typically 5- 
7) of the whole procedure were required for each cluster to obtain a clean, high resolution spherical volume with radius of $5 R_{\mathrm{vir}}$, while keeping the total number of high resolution particles as low as possible. In several cases, additional clusters close to our target had to be included in the high resolution region. Hence, all the initial conditions have at least a spherical volume of radius $5-6 R_{\text {vir }}$ "clean" of low resolution particles, and centered on the target cluster (see Table A1]. The total number of high resolution particles needed is typically only $2-3$ times larger than the number of high resolution particles within this regions of interest. Two of the selected clusters turned out to have a close-by companion with a mass larger than $10^{15} h^{-1} M_{\odot}$. Whereas the 24 targeted clusters represent a fair volume-limited sample of galaxy clusters, the whole simulation sample encompasses in total 26 clusters with masses above $10^{15} h^{-1} M_{\odot}$. In addition, many other clusters with masses between $10^{14} h^{-1} M_{\odot}$ and $10^{15} h^{-1} M_{\odot}$ were found close to our massive targets. 50 of them are free from low-resolution particles up to at least $1 R_{\mathrm{vir}}$. We also extracted initial conditions of 5 more isolated cluster, having masses of $\approx 5,7,4,1,1 \times 10^{14} h^{-1} M_{\odot}$. Such additional clusters are of interest when studying scaling relations (Fabjan et al. 2011).

\section{A2 Adding the baryonic component}

Once the IC for the DM particles have been obtained, the baryonic component was added. The high resolution dark matter particles are splitted into one gas and one DM particle. The mass of the initial DM particle is splitted according to the cosmic baryon fraction, conserving the center of mass and the momentum of the parent DM particle. We displaced them by half the mean interparticle distance. Here we added a further optimization. Taking the "cleaned region" around all clusters of interest within the high resolution region, we traced back the corresponding Lagrangian region into the initial conditions. To associate concave volume to the selected particles, we measured their distance to the center of the high resolution region and calculated the maximum distance found in each direction by sampling the sphere using a HealPIX discretization (Górski et al.2005). Only those dark matter particles which are found within such a volume (including a very small safety buffer) were splitted into one gas and one DM particles. This typically saves $\approx 20 \%$ of gas particles while still having splitted dark matter (and, accordingly, gas particles) within the full extent of the "clean region".

In the left panel of figure A3 in the central part, the spatial extent of the whole high resolution region compared to the extent of baryon-filled region is visible. 


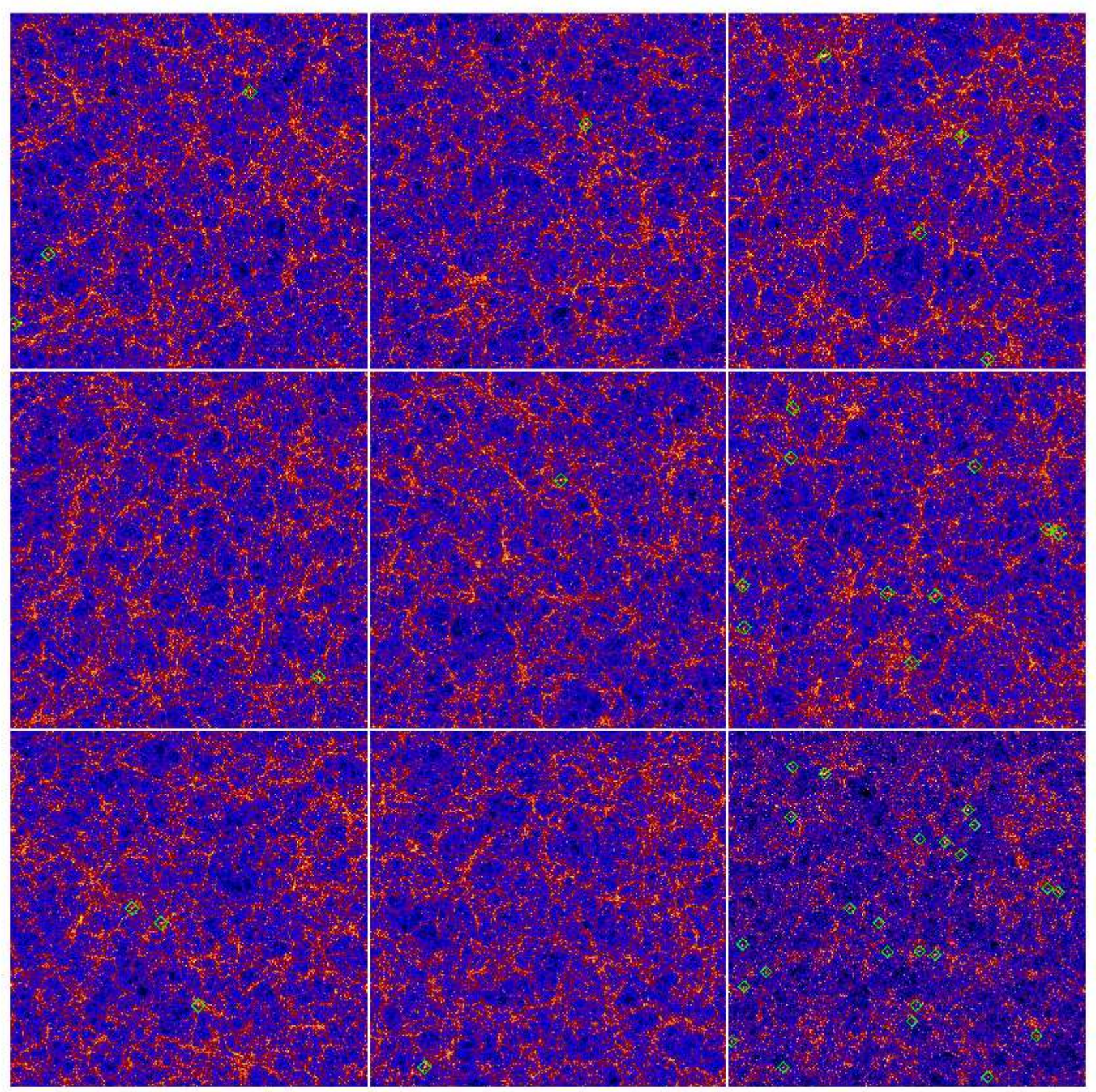

Figure A1. $125 \mathrm{Mpch}^{-1}$ thick slices through the parent DM simulation at $z=0$ showing the projected density. The Diamonds indicate the positions of the 24 most massive clusters extracted for high resolution re-simulations. The bottom right panel shows the projected density through the whole box. 


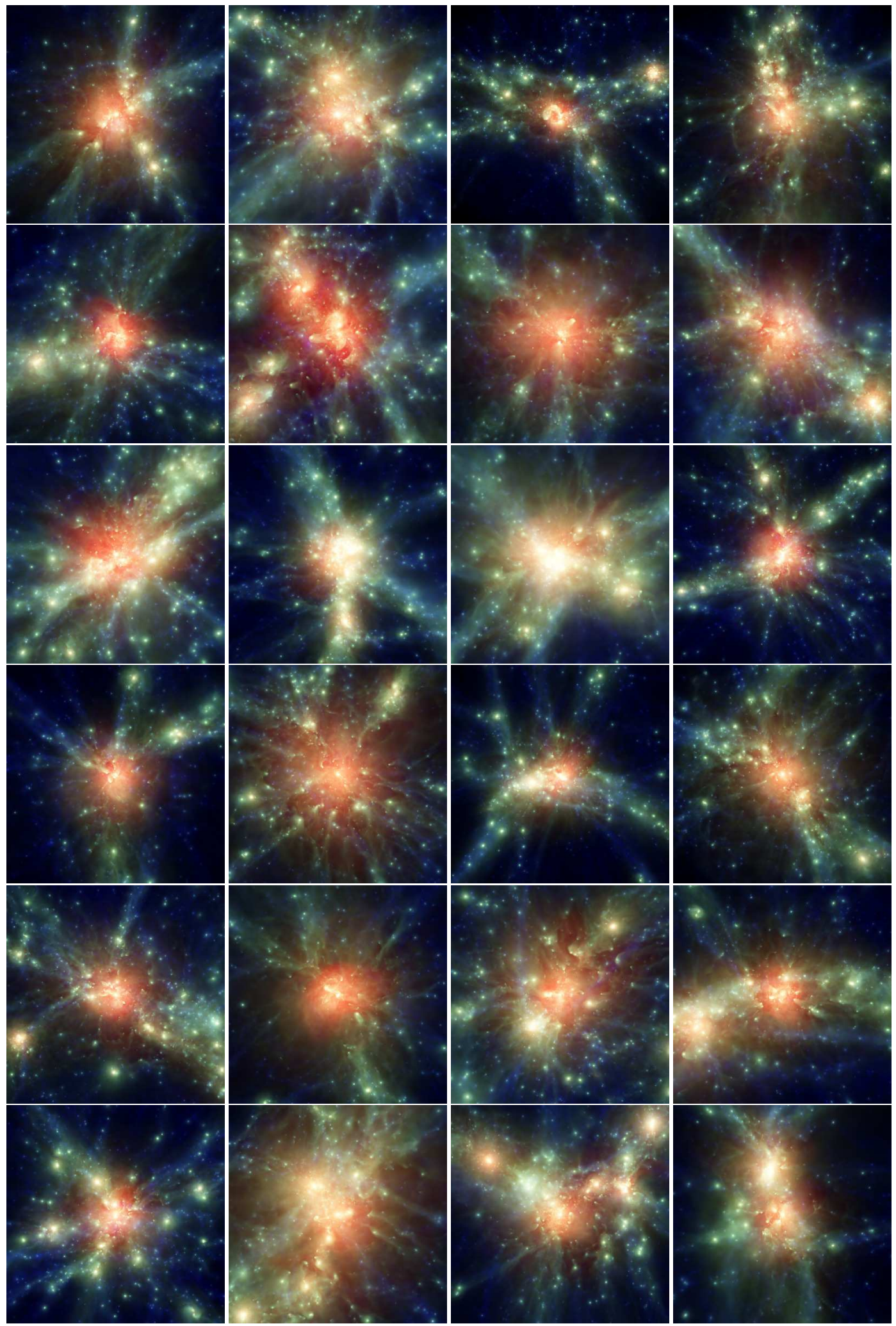

Figure A2. Ray-tracing images of a $15 \mathrm{Mpch}^{-1}$ regions around the center of the individual clusters. Color coded is the temperature of the gas. 
Table A2. Clusters with mass $10^{15} h^{-1} M_{\odot}>M_{\mathrm{vir}}>10^{14} h^{-1} M_{\odot}$ free of low-resolution particles.

\begin{tabular}{|c|c|c|c|c|}
\hline Cluster name & $\begin{array}{c}M_{\text {vir }} \\
M_{\odot} \mathrm{h}^{-1}\end{array}$ & $\begin{array}{c}M_{\text {gas }} \\
h^{-1} M_{\odot}\end{array}$ & $\begin{array}{c}R_{\text {vir }} \\
\mathrm{kpc} \mathrm{h}^{-1}\end{array}$ & $\begin{array}{c}R_{\text {cleaned }} \\
R_{\text {vir }}\end{array}$ \\
\hline d1_9 & $1.436 \mathrm{E}+14$ & $2.067 \mathrm{E}+13$ & 1117.11 & $>5$ \\
\hline d2_2 & $1.708 \mathrm{E}+14$ & $2.506 \mathrm{E}+13$ & 1183.26 & $\geqslant 5$ \\
\hline $\mathrm{d} 2 \_5$ & $1.646 \mathrm{E}+14$ & $2.331 \mathrm{E}+13$ & 1168.81 & $\geqslant 5$ \\
\hline d2_6 & $1.070 \mathrm{E}+14$ & $1.540 \mathrm{E}+13$ & 1013.07 & $\geqslant 5$ \\
\hline d3_4 & $5.249 \mathrm{E}+14$ & $7.466 \mathrm{E}+13$ & 1723.11 & $>5$ \\
\hline d3_23 & $1.219 \mathrm{E}+14$ & $1.709 \mathrm{E}+13$ & 1057.81 & $>5$ \\
\hline d5_2 & $7.707 \mathrm{E}+14$ & $1.104 \mathrm{E}+14$ & 1955.10 & 4 \\
\hline d5_6 & $1.768 \mathrm{E}+14$ & $2.642 \mathrm{E}+13$ & 1197.06 & $\geqslant 5$ \\
\hline d5_11 & $2.266 \mathrm{E}+14$ & $3.327 \mathrm{E}+13$ & 1300.91 & 4 \\
\hline d5_25 & $2.259 \mathrm{E}+14$ & $3.301 \mathrm{E}+13$ & 1299.61 & 4 \\
\hline d6_6 & $1.434 \mathrm{E}+14$ & $2.117 \mathrm{E}+13$ & 1116.47 & $>5$ \\
\hline d6_11 & $2.286 \mathrm{E}+14$ & $3.191 \mathrm{E}+13$ & 1304.73 & $>5$ \\
\hline d6_18 & $1.310 \mathrm{E}+14$ & $1.971 \mathrm{E}+13$ & 1083.39 & 1 \\
\hline d6_26 & $5.762 \mathrm{E}+14$ & $8.107 \mathrm{E}+13$ & 1777.52 & $\geqslant 5$ \\
\hline d7_11 & $1.520 \mathrm{E}+14$ & $2.232 \mathrm{E}+13$ & 1138.18 & $>5$ \\
\hline d8_1 & $4.884 \mathrm{E}+14$ & $7.196 \mathrm{E}+13$ & 1682.05 & 3 \\
\hline d8_6 & $4.993 E+14$ & $6.883 \mathrm{E}+13$ & 1694.58 & $\geqslant 5$ \\
\hline d8_8 & $2.112 \mathrm{E}+14$ & $2.993 \mathrm{E}+13$ & 1270.59 & 2 \\
\hline d8_29 & $1.081 \mathrm{E}+14$ & $1.485 \mathrm{E}+13$ & 1016.29 & $\geqslant 5$ \\
\hline $\mathrm{d} 10 \_2$ & $1.838 \mathrm{E}+14$ & $2.532 \mathrm{E}+13$ & 1212.96 & $\geqslant 5$ \\
\hline d10_3 & $8.074 \mathrm{E}+14$ & $1.131 \mathrm{E}+14$ & 1984.74 & 3 \\
\hline d10_4 & $2.889 \mathrm{E}+14$ & $4.126 \mathrm{E}+13$ & 1411.13 & 3 \\
\hline d10_6 & $1.233 \mathrm{E}+14$ & $1.802 \mathrm{E}+13$ & 1061.86 & $\geqslant 5$ \\
\hline d10_12 & $1.017 \mathrm{E}+14$ & $1.475 \mathrm{E}+13$ & 996.10 & $\geqslant 5$ \\
\hline d10_16 & $1.057 \mathrm{E}+14$ & $1.346 \mathrm{E}+13$ & 1008.81 & $\geqslant 5$ \\
\hline d11_3 & $1.119 \mathrm{E}+14$ & $1.562 \mathrm{E}+13$ & 1028.04 & $\geqslant 5$ \\
\hline d12_1 & $2.622 \mathrm{E}+14$ & $3.596 \mathrm{E}+13$ & 1366.08 & $\geqslant 5$ \\
\hline d12_4 & $3.815 \mathrm{E}+14$ & $5.462 \mathrm{E}+13$ & 1548.76 & $\geqslant 5$ \\
\hline d13_1 & $4.930 \mathrm{E}+14$ & $6.871 \mathrm{E}+13$ & 1687.93 & $\geqslant 5$ \\
\hline d13_2 & $3.808 \mathrm{E}+14$ & $5.518 \mathrm{E}+13$ & 1548.19 & $\geqslant 5$ \\
\hline d13_3 & $4.868 \mathrm{E}+14$ & $7.024 \mathrm{E}+13$ & 1680.78 & 1 \\
\hline d13_7 & $2.426 \mathrm{E}+14$ & $3.830 \mathrm{E}+13$ & 1331.19 & $\geqslant 5$ \\
\hline d14_2 & $1.344 \mathrm{E}+14$ & $2.043 \mathrm{E}+13$ & 1092.54 & $\geqslant 5$ \\
\hline d14_3 & $3.065 \mathrm{E}+14$ & $4.416 \mathrm{E}+13$ & 1439.40 & $\geqslant 5$ \\
\hline d14_5 & $1.754 \mathrm{E}+14$ & $2.519 \mathrm{E}+13$ & 1194.04 & $\geqslant 5$ \\
\hline d15_7 & $1.419 \mathrm{E}+14$ & $2.037 \mathrm{E}+13$ & 1112.67 & $\geqslant 5$ \\
\hline d17_4 & $1.072 \mathrm{E}+14$ & $1.501 \mathrm{E}+13$ & 1013.47 & 1 \\
\hline d18_1 & $2.982 \mathrm{E}+14$ & $4.633 \mathrm{E}+13$ & 1426.32 & $\geqslant 5$ \\
\hline d19_5 & $2.573 \mathrm{E}+14$ & $3.267 \mathrm{E}+13$ & 1357.47 & $\geqslant 5$ \\
\hline $\mathrm{d} 20 \_2$ & $6.489 \mathrm{E}+14$ & $8.920 \mathrm{E}+13$ & 1849.37 & $\geqslant 5$ \\
\hline d20_4 & $1.135 \mathrm{E}+14$ & $1.615 \mathrm{E}+13$ & 1033.07 & $\geqslant 5$ \\
\hline $\mathrm{d} 21 \_2$ & $2.083 \mathrm{E}+14$ & $2.978 \mathrm{E}+13$ & 1264.69 & 4 \\
\hline d21_3 & $3.019 \mathrm{E}+14$ & $4.324 \mathrm{E}+13$ & 1432.09 & 3 \\
\hline d21_19 & $1.031 \mathrm{E}+14$ & $1.687 \mathrm{E}+13$ & 1000.69 & $\geqslant 5$ \\
\hline d21_23 & $1.526 \mathrm{E}+14$ & $2.141 \mathrm{E}+13$ & 1139.66 & $\geqslant 5$ \\
\hline $\mathrm{d} 23 \_2$ & $1.134 \mathrm{E}+14$ & $1.619 \mathrm{E}+13$ & 1032.80 & $\geqslant 5$ \\
\hline $\mathrm{d} 23 \_4$ & $2.970 \mathrm{E}+14$ & $4.217 \mathrm{E}+13$ & 1424.53 & $\geqslant 5$ \\
\hline d23_7 & $1.172 \mathrm{E}+14$ & $1.681 \mathrm{E}+13$ & 1044.22 & $\geqslant 5$ \\
\hline d24_1 & $7.822 \mathrm{E}+14$ & $1.096 \mathrm{E}+14$ & 1964.50 & 3 \\
\hline d24_22 & $1.149 \mathrm{E}+14$ & $1.485 \mathrm{E}+13$ & 1037.26 & $\geqslant 5$ \\
\hline d24_363 & $3.341 \mathrm{E}+14$ & $4.041 \mathrm{E}+12$ & 1479.48 & $\geqslant 5$ \\
\hline
\end{tabular}

Col. 1: Cluster name; Col. 2: Total mass of the cluster inside the virial radius Col. 3: Mass of the gas component inside the virial radius; $\mathrm{Col} 4$ : Virial radius; Col 5: Number of virial radii cleaned by LR particles. 

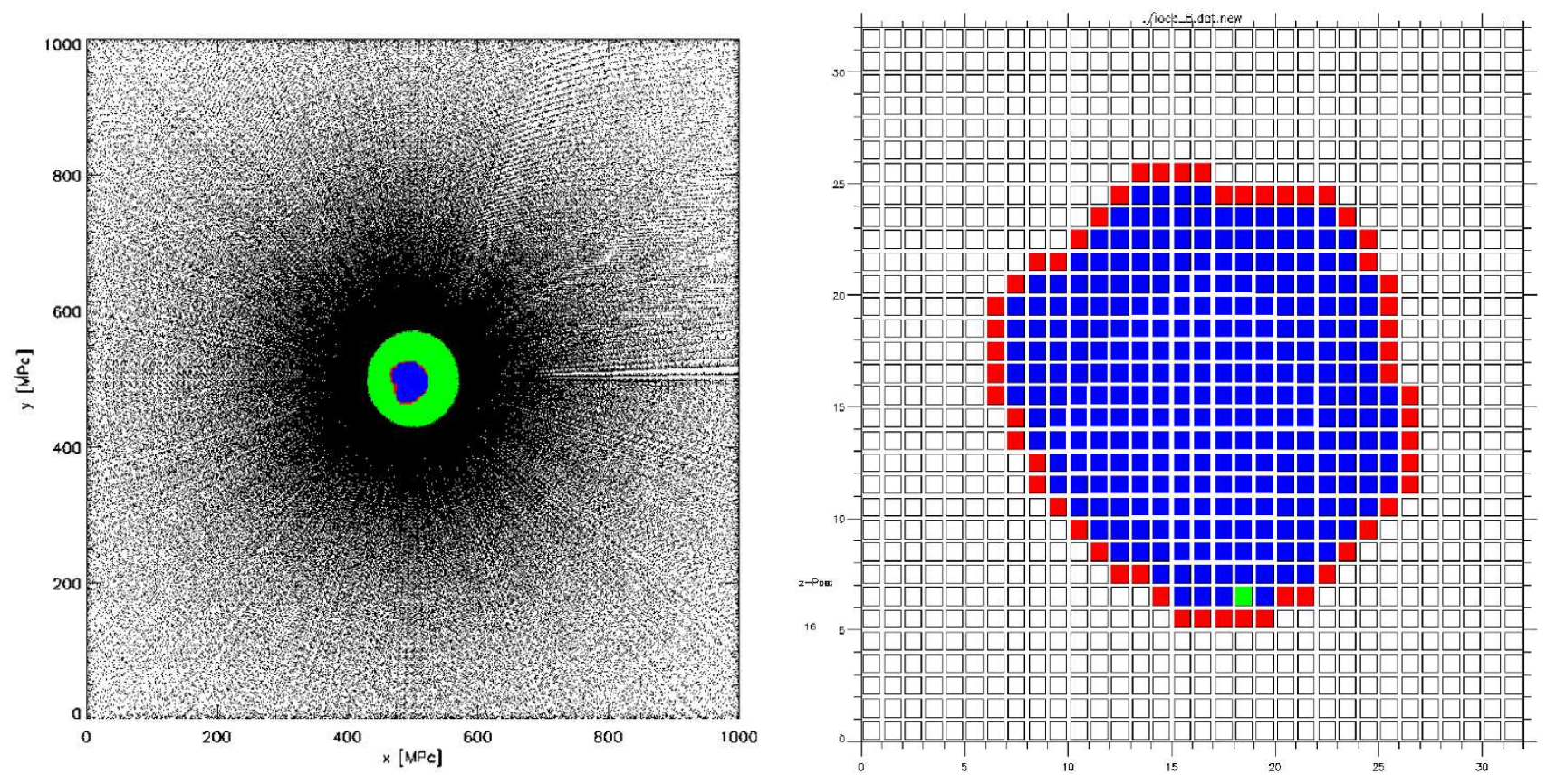

Figure A3. Initial condition region for the high resolution simulations. Left: Black: DM particles with degraded mass resolution outside the HR region, e.g.grained version of the original IC region used in the parent simulation, with increasing mass toward the outer regions. Green: DM particles outside the HR region with the same mass resolution than the parent simulation. This represents a "safety region" where a normal grid is used and particles have the same mass that the parent simulation. Red: HR region. Blue: region where high resolution DM particles have been splitted into gas and DM particles. Right: A slice through the HR initial condition region. Blue boxes refer to the position of the particles traced back, which where at $z=0$ falling within $5 R_{\mathrm{vir}}$ of the target cluster. Red boxes are the cells that are included automatically to obtain a concave region. The green box refer to cell which was added by hand to avoid holes within the HR region. 